\title{
AT2018cow: A Luminous Millimeter Transient
}

\author{
Anna Y. Q. Ho ${ }^{1}$ (1) , E. Sterl Phinney ${ }^{2}$, Vikram Ravi ${ }^{1,3}$ (1), S. R. Kulkarni ${ }^{1}{ }^{(1)}$, Glen Petitpas ${ }^{3}$, Bjorn Emonts ${ }^{4}$, V. Bhalerao ${ }^{5}$ (1), \\ Ray Blundell ${ }^{3}$, S. Bradley Cenko ${ }^{6,7}$ (D), Dougal Dobie ${ }^{8,9}$ (D), Ryan Howie ${ }^{3}$, Nikita Kamraj ${ }^{1}$ (D), Mansi M. Kasliwal ${ }^{1}$ (D), \\ Tara Murphy $^{8}$ (D), Daniel A. Perley ${ }^{10}$ (D) T. K. Sridharan ${ }^{3}$, and Ilsang Yoon ${ }^{4}$ (D) \\ ${ }^{1}$ Cahill Center for Astrophysics, California Institute of Technology, MC 249-17, 1200 E California Boulevard, Pasadena, CA, 91125, USA \\ ${ }^{2}$ Theoretical Astrophysics, MC 350-17, California Institute of Technology, Pasadena, CA 91125, USA \\ ${ }^{3}$ Harvard-Smithsonian Center for Astrophysics, 60 Garden Street, Cambridge, MA 02138, USA \\ ${ }^{4}$ National Radio Astronomy Observatory, 520 Edgemont Road, Charlottesville, VA 22903, USA \\ ${ }^{5}$ Department of Physics, Indian Institute of Technology Bombay, Mumbai 400076, India \\ ${ }^{6}$ Astrophysics Science Division, NASA Goddard Space Flight Center, Mail Code 661, Greenbelt, MD 20771, USA \\ ${ }^{7}$ Joint Space-Science Institute, University of Maryland, College Park, MD 20742, USA \\ ${ }^{8}$ Sydney Institute for Astronomy, School of Physics, University of Sydney, Sydney, New South Wales 2006, Australia \\ ATNF, CSIRO Astronomy and Space Science, P.O. Box 76, Epping, New South Wales 1710, Australia \\ ${ }^{10}$ Astrophysics Research Institute, Liverpool John Moores University, IC2, Liverpool Science Park, 146 Brownlow Hill, Liverpool L3 5RF, UK \\ Received 2018 October 31; revised 2018 November 26; accepted 2018 November 26; published 2019 January 23
}

\begin{abstract}
We present detailed submillimeter- through centimeter-wave observations of the extraordinary extragalactic transient AT2018cow. The apparent characteristics - the high radio luminosity, the rise and long-lived emission plateau at millimeter bands, and the sub-relativistic velocity-have no precedent. A basic interpretation of the data suggests $E_{k} \gtrsim 4 \times 10^{48} \mathrm{erg}$ coupled to a fast but sub-relativistic $(v \approx 0.13 c)$ shock in a dense $\left(n_{e} \approx 3 \times 10^{5} \mathrm{~cm}^{-3}\right)$ medium. We find that the X-ray emission is not naturally explained by an extension of the radio-submm synchrotron spectrum, nor by inverse Compton scattering of the dominant blackbody UV/optical/IR photons by energetic electrons within the forward shock. By $\Delta t \approx 20$ days, the X-ray emission shows spectral softening and erratic inter-day variability. Taken together, we are led to invoke an additional source of X-ray emission: the central engine of the event. Regardless of the nature of this central engine, this source heralds a new class of energetic transients shocking a dense medium, which at early times are most readily observed at millimeter wavelengths.
\end{abstract}

Key words: gamma-ray burst: general - radio continuum: general - submillimeter: general - supernovae: general X-rays: general

\section{Introduction}

\subsection{The Transient Millimeter Sky}

Although the sky is regularly monitored across many bands of the electromagnetic spectrum (as well as in gravitational waves and energetic particles) the dynamic sky at millimeter to submillimeter wavelengths $(0.1-10 \mathrm{~mm})$ remains poorly explored. There has only been one blind transient survey specific to the millimeter band ${ }^{11}$ (Whitehorn et al. 2016); millimeter facilities are usually only triggered after an initial discovery at another wavelength. Even when targeting known transients, the success rate for detection is low, and only a few extragalactic transients ${ }^{12}$ have well-sampled, multifrequency light curves to date. This sample includes supernovae (SNe; Weiler et al. 2007; Horesh et al. 2013), tidal-disruption events (TDEs; Zauderer et al. 2011; Yuan et al. 2016), and gamma-ray bursts (GRBs; de Ugarte Postigo et al. 2012; Laskar et al. 2013; Perley et al. 2014; Urata et al. 2014).

The paucity of millimeter transient studies can be attributed in part to costly receiver and electronics systems and the need for excellent weather conditions, but it also reflects challenges intrinsic to millimeter-wave transients themselves: most known

\footnotetext{
11 The authors searched for transient sources at 90 and $150 \mathrm{GHz}$. They found a single candidate event, which intriguingly showed linear polarization.

12 Here we use "transient" as distinct from "variable": millimeter observations are used to study variability in protostars (e.g., Herczeg et al. 2017) and more commonly for active galactic nuclei (e.g., Dent et al. 1983). There have also been millimeter detections of galactic transient sources, primarily stellar flares (e.g., Bower et al. 2003; Fender et al. 2015).
}

classes are either too dim ( $\mathrm{SNe}$, most TDEs) to detect unless they are very nearby, or too short-lived (GRBs) to detect without very rapid reaction times $(<1$ day, and even in these circumstances the emission may only be apparent from lowdensity environments; Laskar et al. 2013).

An evolving technical landscape, together with rapid followup enabled by high-cadence optical surveys, present new opportunities for millimeter transient astronomy. Lower-noise receivers and ultra-wide bandwidth capability have greatly increased the sensitivity of submm facilities (e.g., the Submillimeter Array or SMA; Ho et al. 2004), and the Atacama Large Millimeter Array (ALMA), a flagship facility, recently began operations. Optical surveys are discovering new and unexpected classes of transient events whose millimeter properties are unknown-and possibly different from previously known types-motivating renewed follow-up efforts.

\subsection{AT2018cow}

AT2018cow was discovered on 2018 June 16 UT as an optical transient (Prentice et al. 2018; Smartt et al. 2018) by the Asteroid Terrestrial-impact Last Alert System (ATLAS; Tonry et al. 2018). It attracted immediate attention because of its fast rise time ( $t_{\text {peak }} \lesssim 3$ days), which was established by earlier non-detections (Fremling 2018; Prentice et al. 2018), together with its high optical luminosity $\left(M_{\text {peak }} \sim-20\right)$ and its close proximity $(d=60 \mathrm{Mpc})$.

UV/optical/IR (UVOIR) observations (Perley et al. 2018; Prentice et al. 2018) revealed unprecedented photometric and spectroscopic properties. Long-lived luminous X-ray emission was detected with the Neil Gehrels Swift Observatory/X-ray 
Telescope (Swift/XRT; Rivera Sandoval \& Maccarone 2018), International Gamma-Ray Astrophysics Laboratory (INTEGRAL) (Ferrigno et al. 2018; Savchenko et al. 2018), and the Nuclear Spectroscopic Telescope Array (NuSTAR) (Grefenstette et al. 2018; Margutti et al. 2018a). Early radio and submillimeter detections were reported by Northern Extended Millimeter Array (NOEMA) (de Ugarte Postigo et al. 2018), JCMT (Smith et al. 2018), Arcminute Microkelvin Imager (AMI) (Bright et al. 2018), and by us using the Australia Telescope Compact Array (ATCA) (Dobie et al. 2018a, 2018b). The source does not appear to be a GRB, as no prompt high-energy emission was detected in searches of Swift/Burst Alert Telescope (BAT) (Lien et al. 2018), Fermi/ GBM (Dal Canton et al. 2018), Fermi/Large Area Telescope (LAT) (Kocevski \& Cheung 2018), and AstroSat Cadmium Zinc Telluride Imager (CZTI) (Sharma et al. 2018).

Perley et al. (2018) suggested that AT2018cow is a new member of the class of rapidly rising ( $t_{\text {rise }} \lesssim 5$ days $)$ and luminous $\left(M_{\text {peak }}<-18\right)$ blue transients, which have typically been found in archival searches of optical surveys (Drout et al. 2014; Pursiainen et al. 2018; Rest et al. 2018). The leading hypothesis for this class was circumstellar interaction of a supernova (SN; Ofek et al. 2010), but this was difficult to test because most of the events were located at cosmological distances, and not discovered in real time. AT2018cow presented the first opportunity to study a member of this class up close and in real time, but its origin remains mysterious despite the intense ensuing observational campaign. Possibilities include failed SNe and TDEs, but although AT2018cow shares properties with both of these classes, it is clearly not a typical member of either (Kuin et al. 2018; Perley et al. 2018; Prentice et al. 2018).

Given the unusual nature of the source, we were motivated to undertake high-frequency observations. We began an extensive monitoring campaign with the SMA at 230 and $340 \mathrm{GHz}$ and carried out supporting observations with the ATCA from 5-34 GHz. To our surprise AT2018cow was very bright and still rising at submillimeter wavelengths (and optically thick in the centimeter band) days after the discovery. Our SMA observations represent the first millimeter observation of a transient in its rise phase.

This finding led us to seek Director's Discretionary Time (DDT) with ALMA at even higher frequencies, which enabled us to resolve the peak of the spectral energy distribution (SED). A technical highlight of the ALMA observations was the detection of the source at nearly a terahertz frequency (Band 9). We present the submillimeter, radio, and X-ray observations in Section 2, and our modeling of the radio-emitting ejecta in Section 3. In Section 4, we put our velocity and energy measurements in the context of other transients (Section 4.1), attribute the high submm luminosity of AT2018cow to the large density of the surrounding medium (Section 4.2), and discuss some problems with the synchrotron model parameters (Section 4.3). In Section 5, we attribute the late-time X-ray emission to a powerful central engine. We look ahead to the future in Section 6.

\section{Observations}

All observations are measured $\Delta t$ (observer frame) from the zero-point MJD 58285 (following Perley et al. 2018), which lies between the date of discovery (MJD 58285.441) and the last non-detection (58284.13; Prentice et al. 2018). The full set of flux-density measurements at radio and millimeter wavelengths is presented in Table 1. At $\Delta t=14$ days we find excellent agreement between the SMA and the ALMA data, showing that the flux scales are consistent.

\subsection{Radio and Submillimeter Observations}

\subsubsection{The SMA}

AT2018cow was regularly observed with the SMA under its DDT/Target of opportunity program. Observations took place over the period of UT 2018 June 21-UT 2018 August 3 ( $\Delta t \approx 5-49$ days) in the Compact configuration, with an additional epoch on UT 2018 August 31 ( $\Delta t \approx 76$ days). All observations contained 6-8 antennas and covered a range of baseline lengths from $16.4-77 \mathrm{~m}$. A majority of these observations were short and were repeated almost nightly by sharing tuning and calibration data with other science tracks. The SMA has two receiver sets each with $8 \mathrm{GHz}$ of bandwidth in each of two sidebands $(32 \mathrm{GHz}$ total), covering a range of frequencies from $188-416 \mathrm{GHz}$. Each receiver can be tuned independently to provide dual-band observations. Additionally, the upper and lower sidebands are separated (center to center) by $16 \mathrm{GHz}$, allowing up to four simultaneous frequency measurements. During some observations, the receivers were tuned to the same local oscillator frequency, allowing the lower and upper sidebands to be averaged together, improving the signal-to-noise ratio. For all observations, the quasars 1635 +381 and 3C 345 were used as primary phase and amplitude gain calibrators, respectively, with absolute flux calibration performed by nightly comparison to Titan, Neptune, or (maserfree) continuum observations of the emission-line star MWC349a. The quasar 3C 279 and/or the blazar 3C 454.3 was used for bandpass calibration. Data were calibrated in IDL using the MIR package. Additional analysis and imaging were performed using the MIRIAD package. Given that the target was a point source, fluxes were derived directly from the calibrated visibilities, but the results agree well with flux estimates derived from the CLEANed images when the data quality and UV coverage was adequate.

\subsubsection{The ATCA}

We obtained six epochs of centimeter-wavelength observations with the ATCA (Frater et al. 1992). During the first three epochs, the six $22 \mathrm{~m}$ dishes were arranged in an east-west $1.5 \mathrm{~A}$ configuration, with baselines ranging from $153-4469 \mathrm{~m}$. During the latter three epochs, five of the six dishes were moved to a compact $\mathrm{H} 75^{13}$ configuration, occupying a cardinally oriented "T" with baselines ranging from 31-89 m. Full-Stokes data were recorded with the Compact Array Broadband Backend (Wilson et al. 2011) in a standard continuum CABB Filter Bank (CFB) $1 M$ setup, simultaneously providing two $2.048 \mathrm{GHz}$ bands each with 2048 channels. Observations were obtained with center frequencies of 5.5 and $9 \mathrm{GHz}, 16.7$ and $21.2 \mathrm{GHz}$, and 33 and $35 \mathrm{GHz}$, with data in the latter two bands typically being averaged to form a band centered at $34 \mathrm{GHz}$. The flux-density scale was set using observations of the ATCA flux standard PKS 1934-638. For observations below $33 \mathrm{GHz}$, PKS 1934-638 was also used to calibrate the complex time-independent bandpasses, and

\footnotetext{
13 https://www.narrabri.atnf.csiro.au/operations/array_configurations/ configurations.html
} 
regular observations of the compact quasar PKS $1607+268$ were used to calibrate the time-variable complex gains. For the higher-frequency observations, a brighter source (3C 279) was used for bandpass calibration (except for epochs 1 and 4), and the compact quasar 4C 10.45 was used for gain calibration. In the $\mathrm{H} 75$ configuration, we only report results from observations at $34 \mathrm{GHz}$, from baselines not subject to antenna shadowing. For all $34 \mathrm{GHz}$ observations, data obtained with the sixth antenna located $4500 \mathrm{~m}$ from the center of the array were discarded because of the difficulty of tracking the differential atmospheric phase over the long baselines to this antenna. The weather was good for all observations, with negligible wind and $<500 \mu \mathrm{m}$ of $\mathrm{rms}$ atmospheric path-length variations (Middelberg et al. 2006).

The data were reduced and calibrated using standard techniques implemented in the MIRIAD software (Sault et al. 1995). To search for unresolved emission at the position of AT2018cow, we made multifrequency synthesis images with uniform weighting. Single rounds of self-calibration over 5-10 minute intervals were found to improve the image quality in all bands. For data at 5.5 and $9 \mathrm{GHz}$, point-source models of all strong unresolved field sources were used for self-calibration. For data at the higher frequencies, selfcalibration was performed using a point-source model for AT2018cow itself, as no other sources were detected within the primary beams, and AT2018cow was detected with a sufficient signal-to-noise ratio. We report flux densities derived by fitting point-source models to the final images using the MIRIAD task imfit.

\subsubsection{ALMA}

AT2018cow was observed with ALMA as part of DDT during Cycle 5 using Bands 3, 4, 7, 8, and 9. Observations were performed on 2018 June 30 ( $\Delta t \approx 14$ days; Bands 7 and 8 ), 2018 July 08 ( $\Delta t \approx 22$ days; Bands 3 and 4 ), and on 2018 July 10 ( $\Delta t \approx 23$ days; Band 9$){ }^{14}$

The ALMA $12 \mathrm{~m}$ antenna array was in its most compact C43-1 configuration, with 46-48 working antennas and baselines ranging from $12-312 \mathrm{~m}$. The on-source integration time was 6-8 minutes for Bands $3-8$, and 40 minutes for Band 9. The Band 3-8 observations used two-sideband (2SB) receivers with $4 \mathrm{GHz}$ bandwidth each centered on 91.5 and 103.5 GHz (Band 3), 138 and $150 \mathrm{GHz}$ (Band 4), 337.5 and $349.5 \mathrm{GHz}$ (Band 7), and 399 and $411 \mathrm{GHz}$ (Band 8). The Band 9 observations used double-sideband (DSB) receivers with $8 \mathrm{GHz}$ bandwidth (2 times larger than that for the Band 3-8 observation, by using $90^{\circ}$ Walsh phase switching) centered on 663 and $679 \mathrm{GHz}$. All calibration and imaging was done with the Common Astronomical Software Applications (CASA; McMullin et al. 2007). The data in Bands 3-8 were calibrated with the standard ALMA pipeline, using J1540 +1447 , J1606+1814, or J1619+2247 to calibrate the complex gains, and using J1337-1257 (Band 7), J1550+0527 (Band 3/4), or J1517-2422 (Band 8) to calibrate the bandpass response and apply an absolute flux scale. Band 9 observations were delivered following manual calibration by the North American ALMA Science Center, using $\mathrm{J} 1540+1447$ for gain calibration, and J1517-2422 for bandpass- and flux calibration. We subsequently applied a phase-only self-calibration using the target source

\footnotetext{
$\overline{14}$ Band 9 observations were also performed on 2018 July 09, but these data were of too poor quality to use as a result of weather conditions.
}

Table 1

Flux-density Measurements for AT2018cow

\begin{tabular}{|c|c|c|c|}
\hline$\Delta t$ (days) & Facility & Frequency $(\mathrm{GHz})$ & Flux Density (mJy) \\
\hline 5.39 & SMA & 215.5 & $15.14 \pm 0.56$ \\
\hline 5.39 & SMA & 231.5 & $16.19 \pm 0.65$ \\
\hline 6.31 & SMA & 215.5 & $31.17 \pm 0.87$ \\
\hline 6.31 & SMA & 231.5 & $31.36 \pm 0.97$ \\
\hline 7.37 & SMA & 215.5 & $40.19 \pm 0.56$ \\
\hline 7.37 & SMA & 231.5 & $41.92 \pm 0.66$ \\
\hline 7.41 & SMA & 330.8 & $36.39 \pm 2.25$ \\
\hline 7.41 & SMA & 346.8 & $30.7 \pm 1.99$ \\
\hline 8.37 & SMA & 215.5 & $41.19 \pm 0.47$ \\
\hline 8.37 & SMA & 231.5 & $41.44 \pm 0.56$ \\
\hline 8.38 & SMA & 344.8 & $26.74 \pm 1.42$ \\
\hline 8.38 & SMA & 360.8 & $22.79 \pm 1.63$ \\
\hline 9.26 & SMA & 243.3 & $35.21 \pm 0.75$ \\
\hline 9.26 & SMA & 259.3 & $36.1 \pm 1.0$ \\
\hline 9.28 & SMA & 341.5 & $22.85 \pm 1.53$ \\
\hline 9.28 & SMA & 357.5 & $25.84 \pm 2.5$ \\
\hline 10.26 & SMA & 243.3 & $36.6 \pm 0.81$ \\
\hline 10.26 & SMA & 259.3 & $31.21 \pm 0.92$ \\
\hline 10.26 & SMA & 341.5 & $19.49 \pm 1.47$ \\
\hline 10.26 & SMA & 357.5 & $17.42 \pm 2.8$ \\
\hline 11.26 & SMA & 243.3 & $22.14 \pm 1.05$ \\
\hline 11.26 & SMA & 259.3 & $20.02 \pm 1.28$ \\
\hline 13.3 & SMA & 215.5 & $35.67 \pm 0.81$ \\
\hline 13.3 & SMA & 231.5 & $32.94 \pm 1.01$ \\
\hline 14.36 & SMA & 344.8 & $26.85 \pm 2.22$ \\
\hline 14.36 & SMA & 360.8 & $26.13 \pm 2.77$ \\
\hline 14.37 & SMA & 215.5 & $42.05 \pm 0.5$ \\
\hline 14.37 & SMA & 231.5 & $38.71 \pm 0.58$ \\
\hline 15.23 & SMA & 225.0 & $30.82 \pm 2.41$ \\
\hline 15.23 & SMA & 233.0 & $28.64 \pm 4.0$ \\
\hline 15.23 & SMA & 241.0 & $27.41 \pm 3.21$ \\
\hline 15.23 & SMA & 249.0 & $15.4 \pm 4.74$ \\
\hline 17.29 & SMA & 234.6 & $36.57 \pm 1.55$ \\
\hline 17.29 & SMA & 250.6 & $34.04 \pm 1.81$ \\
\hline 18.4 & SMA & 217.5 & $52.52 \pm 0.55$ \\
\hline 18.4 & SMA & 233.5 & $49.32 \pm 0.65$ \\
\hline 19.25 & SMA & 193.5 & $59.27 \pm 1.49$ \\
\hline 19.25 & SMA & 202.0 & $56.03 \pm 1.5$ \\
\hline 19.25 & SMA & 209.5 & $55.09 \pm 1.39$ \\
\hline 19.25 & SMA & 218.0 & $54.54 \pm 1.33$ \\
\hline 20.28 & SMA & 215.5 & $50.6 \pm 1.69$ \\
\hline 20.28 & SMA & 231.5 & $49.16 \pm 1.84$ \\
\hline 20.28 & SMA & 267.0 & $41.69 \pm 1.62$ \\
\hline 20.28 & SMA & 283.0 & $37.84 \pm 1.63$ \\
\hline 24.39 & SMA & 215.5 & $55.57 \pm 0.53$ \\
\hline 24.39 & SMA & 231.5 & $53.2 \pm 0.6$ \\
\hline 24.4 & SMA & 333.0 & $23.98 \pm 1.39$ \\
\hline 24.4 & SMA & 349.0 & $28.46 \pm 1.37$ \\
\hline 26.26 & SMA & 215.6 & $38.83 \pm 1.2$ \\
\hline 26.26 & SMA & 231.6 & $34.1 \pm 1.33$ \\
\hline 31.2 & SMA & 230.6 & $36.76 \pm 1.12^{\mathrm{a}}$ \\
\hline 31.2 & SMA & 246.6 & $31.41 \pm 1.42^{\mathrm{a}}$ \\
\hline 35.34 & SMA & 215.5 & $21.59 \pm 0.89$ \\
\hline 35.34 & SMA & 231.5 & $20.63 \pm 1.04$ \\
\hline 36.34 & SMA & 215.5 & $24.32 \pm 1.19$ \\
\hline 36.34 & SMA & 231.5 & $20.79 \pm 1.42$ \\
\hline 39.25 & SMA & 217.0 & $18.34 \pm 1.65$ \\
\hline 39.25 & SMA & 233.0 & $19.74 \pm 1.76$ \\
\hline 39.26 & SMA & 264.0 & $17.61 \pm 2.79$ \\
\hline 39.26 & SMA & 280.0 & $8.27 \pm 2.93$ \\
\hline 41.24 & SMA & 217.0 & $12.58 \pm 1.5$ \\
\hline 41.24 & SMA & 225.0 & $8.91 \pm 1.9$ \\
\hline 41.24 & SMA & 233.0 & $15.08 \pm 1.73$ \\
\hline 41.24 & SMA & 241.0 & $9.64 \pm 2.13$ \\
\hline 44.24 & SMA & 230.6 & $9.42 \pm 1.61$ \\
\hline
\end{tabular}


Table 1

(Continued)

\begin{tabular}{|c|c|c|c|}
\hline$\Delta t$ (days) & Facility & Frequency $(\mathrm{GHz})$ & Flux Density (mJy) \\
\hline 44.24 & SMA & 234.6 & $8.04 \pm 2.51$ \\
\hline 44.24 & SMA & 246.3 & $10.43 \pm 2.13$ \\
\hline 44.24 & SMA & 250.6 & $10.06 \pm 3.24$ \\
\hline 45.23 & SMA & 217.0 & $8.28 \pm 2.24$ \\
\hline 45.23 & SMA & 233.0 & $10.55 \pm 2.39$ \\
\hline 45.23 & SMA & 264.0 & $8.35 \pm 3.27$ \\
\hline 45.23 & SMA & 280.0 & $5.7 \pm 3.49$ \\
\hline 47.24 & SMA & 230.6 & $11.47 \pm 2.81$ \\
\hline 47.24 & SMA & 234.6 & $10.81 \pm 4.39$ \\
\hline 47.24 & SMA & 246.6 & $11.65 \pm 3.76$ \\
\hline 47.24 & SMA & 250.6 & $5.6 \pm 5.37$ \\
\hline 48.31 & SMA & 217.5 & $7.63 \pm 1.11$ \\
\hline 48.31 & SMA & 233.5 & $5.73 \pm 1.32$ \\
\hline 76.27 & SMA & 215.5 & $1.33 \pm 0.55$ \\
\hline 76.27 & SMA & 231.5 & $0.61 \pm 0.63$ \\
\hline 76.27 & SMA & 335.0 & $-2.27 \pm 1.87$ \\
\hline 76.27 & SMA & 351.0 & $-0.32 \pm 1.76$ \\
\hline 10.48 & ATCA & 5.5 & $<0.15$ \\
\hline 10.48 & ATCA & 9.0 & $0.27 \pm 0.06$ \\
\hline 10.48 & ATCA & 34.0 & $5.6 \pm 0.16$ \\
\hline 13.47 & ATCA & 5.5 & $0.22 \pm 0.05$ \\
\hline 13.47 & ATCA & 9.0 & $0.52 \pm 0.04$ \\
\hline 13.47 & ATCA & 16.7 & $1.5 \pm 0.1$ \\
\hline 13.47 & ATCA & 21.2 & $2.3 \pm 0.3$ \\
\hline 13.47 & ATCA & 34.0 & $7.6 \pm 0.5$ \\
\hline 17.47 & ATCA & 5.5 & $0.41 \pm 0.04$ \\
\hline 17.47 & ATCA & 9.0 & $0.99 \pm 0.03$ \\
\hline 19.615 & ATCA & 34.0 & $14.26 \pm 0.21$ \\
\hline 28.44 & ATCA & 34.0 & $30.59 \pm 0.2$ \\
\hline 34.43 & ATCA & 34.0 & $42.68 \pm 0.19$ \\
\hline 81.37 & ATCA & 34.0 & $6.97 \pm 0.09$ \\
\hline 14.03 & ALMA & 336.5 & $29.4 \pm 2.94$ \\
\hline 14.03 & ALMA & 338.5 & $29.1 \pm 2.91$ \\
\hline 14.03 & ALMA & 348.5 & $28.49 \pm 2.85$ \\
\hline 14.03 & ALMA & 350.5 & $28.29 \pm 2.83$ \\
\hline 14.14 & ALMA & 398.0 & $26.46 \pm 2.65$ \\
\hline 14.14 & ALMA & 400.0 & $26.21 \pm 2.62$ \\
\hline 14.14 & ALMA & 410.0 & $25.69 \pm 2.57$ \\
\hline 14.14 & ALMA & 412.0 & $25.95 \pm 2.6$ \\
\hline 22.02 & ALMA & 90.5 & $91.18 \pm 4.6$ \\
\hline 22.02 & ALMA & 92.5 & $92.31 \pm 4.6$ \\
\hline 22.02 & ALMA & 102.5 & $93.97 \pm 4.7$ \\
\hline 22.02 & ALMA & 104.5 & $93.57 \pm 4.7$ \\
\hline 22.04 & ALMA & 138.0 & $85.1 \pm 4.3$ \\
\hline 22.04 & ALMA & 140.0 & $84.58 \pm 4.2$ \\
\hline 22.04 & ALMA & 150.0 & $80.62 \pm 4.0$ \\
\hline 22.04 & ALMA & 152.0 & $79.71 \pm 4.0$ \\
\hline 23.06 & ALMA & 671.0 & $31.5 \pm 6.3$ \\
\hline
\end{tabular}

Notes. Time of detection used is mean UT of observation. SMA measurements have formal uncertainties shown, which are appropriate for in-band measurements on a given night. However, for night-to-night comparisons, true errors are dominated by systematics and are roughly $10 \%-15 \%$ unless indicated otherwise. ALMA measurements have roughly $5 \%$ uncertainties in Bands 3 and 4, 10\% uncertainties in Bands 7 and 8, and a 20\% uncertainty in Band 9. ATCA measurements have formal errors listed, but also have systematic uncertainties of roughly $10 \%$.

${ }^{a}$ Systematic uncertainty $20 \%$ due to uncertain flux calibration.

(for Bands 3-8), performed a deconvolution, imaged the data, and flux-corrected for the response of the primary beam. AT2018cow is unresolved in our ALMA data, with a synthesized beam that ranges from 3 ! $3 \times 22^{\prime \prime} 5\left(\mathrm{PA}=29^{\circ}\right)$ in Band 3 to
0. . $50 \times 0$. " $36\left(\mathrm{PA}=-46^{\circ}\right)$ in Band 9. The signal-to-noise ratio in the resulting images ranges from $\sim 500$ in Bands 3 and 4 to $\sim 80$ in Band 9. Details about the ALMA Band 9 data reduction can be found in Appendix A.

\subsection{X-Ray Observations}

\subsubsection{Swift/XRT}

Swift (Gehrels et al. 2004) has been monitoring AT2018cow since June 19, with both the Ultraviolet-Optical Telescope (Roming et al. 2005) and the XRT (Burrows et al. 2005). The transient was well detected in both instruments (e.g., Rivera Sandoval et al. 2018).

We downloaded the Swift/XRT data products (light curves and spectra) using the web-based tools developed by the SwiftXRT team (Evans et al. 2009). We used the default values, but binned the data by observation. To convert from count rate to flux, we used the absorbed count-to-flux rate set by the spectrum on the same tool, $4.26 \times 10^{-11} \mathrm{erg} \mathrm{cm}^{-2} \mathrm{ct}^{-1}$. This assumes a photon index of $\Gamma=1.54$ and a Galactic $N_{H}$ column of $6.57 \times 10^{20} \mathrm{~cm}^{-2}$.

\subsubsection{NuSTAR}

NUSTAR (Harrison et al. 2013) comprises two co-aligned telescopes, Focal Plane Module A (FPMA) and FPMB. Each is sensitive to $\mathrm{X}$-rays in the $3-79 \mathrm{keV}$ range, with slightly different response functions. NuSTAR observed AT2018cow on four epochs, and a log of these observations as well as the bestfit spectral model parameters is presented in Table 2.

NUSTAR data were extracted using nustardas_ 06Jul17_v1 from HEASOFT 6.24. Source photons were extracted from a circle of $60^{\prime \prime}$ radius, visually centered on the object. We note that such a large region, appropriate for NUSTAR data, includes the transient as well as the host galaxy. Background photons were extracted from a non-overlapping circular region with $120^{\prime \prime}$ radius on the same chip. Spectra were grouped to 20 source photons per bin, ignoring energies below $3 \mathrm{keV}$ and above $80 \mathrm{keV}$.

Spectra were analyzed in XSPEC (v12.10.0c), using NuSTAR CALDB files dated 2018 August 14. Rivera Sandoval et al. (2018) report a low absorbing column density $\left(N_{\mathrm{H}}=7.0 \times 10^{20} \mathrm{~cm}^{-2}\right)$, hence we ignore this component in fitting. We opt for a simple phenomenological model to describe the spectrum. We do not fit for a cross-normalization constant between NUSTAR FPMA and FPMB. Epoch 1 (OBSID 90401327002) spectra are not consistent with a simple power law or a broken power law, hence we fit it with the bkn2pow model (obtaining spectral breaks at $9.0 \pm 0.3 \mathrm{keV}$ and $11.1 \pm 0.3 \mathrm{keV}$ ). Spectra of the remaining three epochs are well fit by a simple, unabsorbed power law.

We calculate the flux directly from energies of individual source and background photons detected, converted into flux using the Ancillary Response Files generated by the NuSTAR pipeline. We use a bootstrap method to estimate the error bars: we draw photons from the data with replacement, and calculate the source flux from this random sample. By repeating this process 10,000 times for each OBSID and each energy range, we calculate the $1 \sigma$ error bars on the fluxes. This method gives answers consistent with xspec flux and cflux measurements for bright sources (see for instance Kaspi et al. 2014), but has the advantage of giving flux measurements without the need to assume a spectral model for the source. We find that the source 


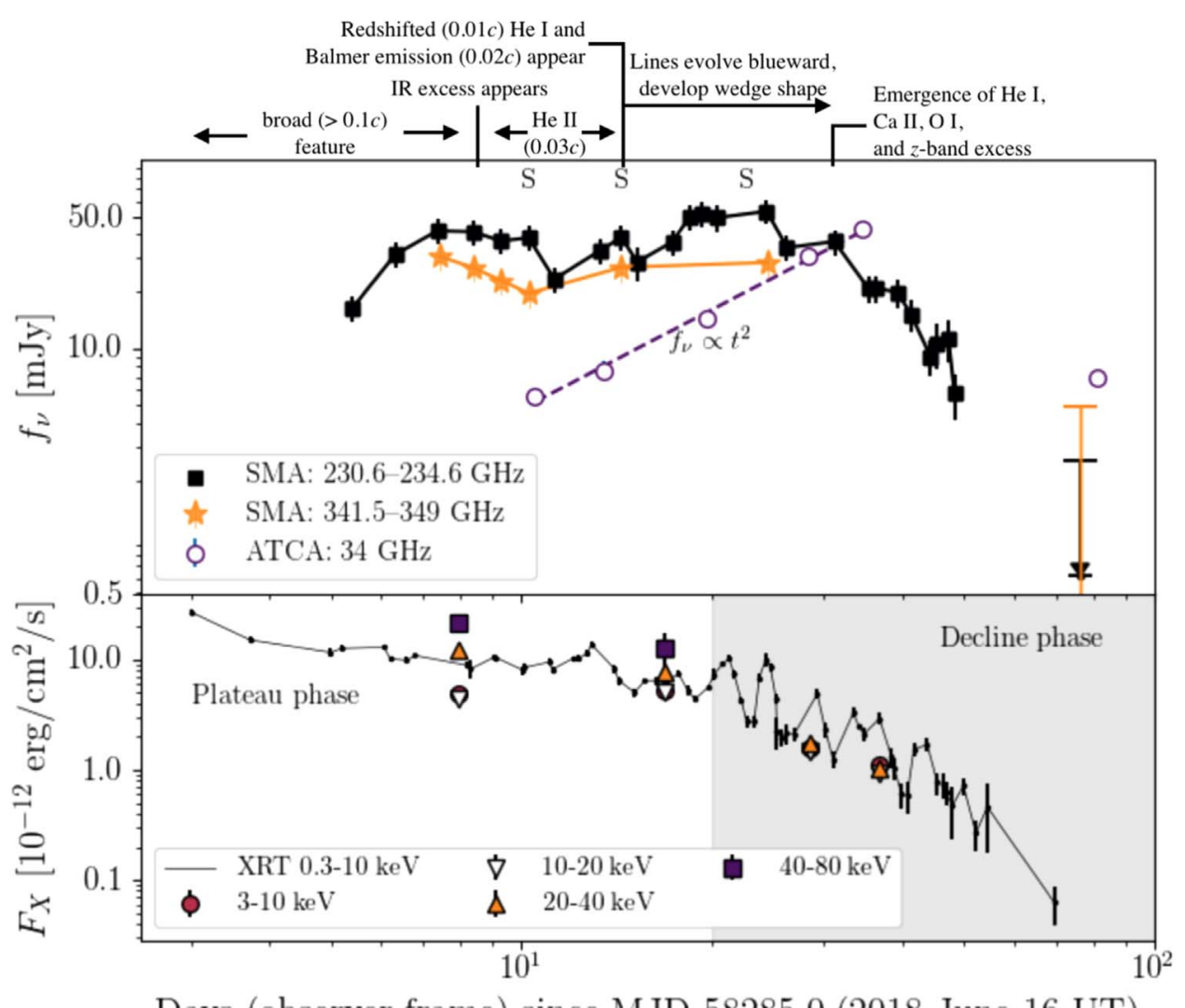

Figure 1. (Top panel) Submillimeter (SMA) through radio (ATCA) light curves of AT2018cow, with a timeline of the evolution of the UVOIR spectra (based on Perley et al. 2018) shown above. There were four SMA observations with no frequency tunings in the ranges shown. For these, we took the closest value to $231.5 \mathrm{GHz}$ (243.3 GHz for Days 9, 10, and $11 ; 218 \mathrm{GHz}$ for Day 19) and scaled them to $231.5 \mathrm{GHz}$ assuming a spectral index $F_{\nu} \propto \nu^{-1}$. We scaled all SMA fluxes so that the reference quasar $1635+381$ would have the value of its mean flux at that frequency. The uncertainties shown on the SMA data represent a combination of formal uncertainties and $15 \%$ systematic uncertainties, which is a conservative estimate. Non-detections are represented as a $3 \sigma$ upper limit (horizontal bar) and a vertical arrow down to the measurement. The upper limit measurement at $350.1 \mathrm{GHz}$ is -0.32 , below the limit of the panel. The error bars shown on the ATCA data are a combination of formal uncertainties and an estimated 10\% systematic uncertainty. The ATCA $34 \mathrm{GHz}$ measurements rise as $t^{2}$, shown as a dotted line. The full set of SMA light curves for all frequency tunings are shown in Appendix B. The letters "S" on the top demarcate the epochs with spectra shown in Figure 3. (Bottom panel) $\mathrm{X}$-ray light curve from Swift/XRT together with four epochs of NuSTAR observations. The last two NuSTAR epochs have a non-detection in the highest-frequency band $(40-80 \mathrm{keV})$. We denote two distinct phases of the X-ray light curve, the plateau phase, and the decline phase, discussed in detail in Section 3.

Table 2

NuSTAR Flux Measurements for AT2018cow and the Spectral Model Parameters

\begin{tabular}{|c|c|c|c|c|c|c|c|c|c|}
\hline \multirow{2}{*}{ Epoch } & \multirow{2}{*}{ OBSID } & \multirow{2}{*}{ Exp. Time (ks) } & \multirow{2}{*}{$\Delta t$ (days) } & \multicolumn{4}{|c|}{ Flux $\left(10^{-12} \mathrm{erg} \mathrm{cm}^{-2} \mathrm{~s}^{-1}\right)$} & \multirow{2}{*}{ Photon Index } & \multirow{2}{*}{$\chi^{2} / \mathrm{DOF}$} \\
\hline & & & & $3-10 \mathrm{keV}$ & $10-20 \mathrm{keV}$ & $20-40 \mathrm{keV}$ & $40-80 \mathrm{keV}$ & & \\
\hline$\overline{1}$ & $90401327002^{\mathrm{a}}$ & 32.4 & 7.9 & $4.94 \pm 0.04$ & $4.41 \pm 0.10$ & $12.21 \pm 0.39$ & $21.46 \pm 4.29$ & $\ldots$ & $421 / 443$ \\
\hline 2 & 90401327004 & 30.0 & 16.8 & $5.21 \pm 0.04$ & $4.99 \pm 0.10$ & $7.70 \pm 0.33$ & $12.80 \pm 4.79$ & $1.39 \pm 0.02$ & $424 / 412$ \\
\hline 3 & 90401327006 & 31.2 & 28.5 & $1.58 \pm 0.03$ & $1.45 \pm 0.06$ & $1.74 \pm 0.21$ & $\cdots$ & $1.51 \pm 0.04$ & $174 / 169$ \\
\hline 4 & 90401327008 & 33.0 & 36.8 & $1.10 \pm 0.02$ & $0.92 \pm 0.05$ & $1.02 \pm 0.20$ & $\ldots$ & $1.59 \pm 0.05$ & $134 / 135$ \\
\hline
\end{tabular}

Notes. Fluxes were measured with a model-independent method.

${ }^{\mathrm{a}}$ OBSID 90401327002 is best described by a bkn2pow model with parameters $\Gamma_{1}=1.24 \pm 0.05, E_{1}=9.0 \pm 0.3 \mathrm{keV}, \Gamma_{2}=3.6 \pm 0.7, E_{2}=11.1 \pm 0.3 \mathrm{keV}$, $\Gamma_{3}=0.50 \pm 0.05$. All reported values are for this model.

is not well detected in the $40-80 \mathrm{keV}$ band at the third and fourth epochs.

\section{Basic Properties of the Shock}

\subsection{Light Curve}

The radio and X-ray light curves are shown in Figure 1. The $230 \mathrm{GHz}$ light curve rises (the first observation of a millimeter transient in its rise phase) and then shows significant variability, presumably from inhomogeneities in the surrounding medium. We have tentative evidence that the rise is at least in part due to a decreasing peak frequency: at $\Delta t=5-6$ days, the flux is marginally higher at $231.5 \mathrm{GHz}$ than at $215.5 \mathrm{GHz}$, and at $\Delta t=7-8$ days, it seems that the peak may have been within the SMA observing bands. However, the position of the peak is ill-constrained; future early observations would benefit from observations at more frequencies. 

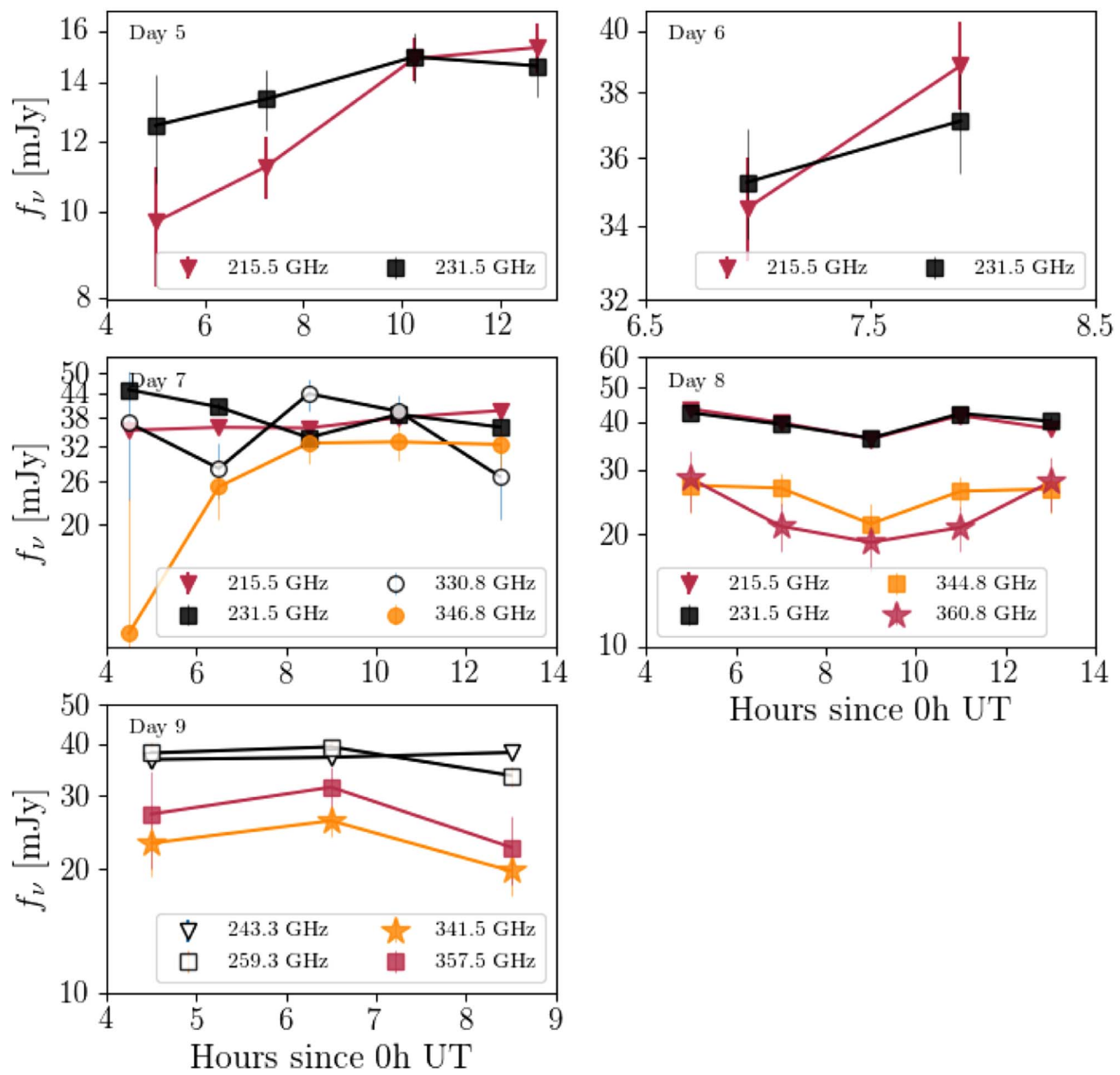

Figure 2. Zoomed-in light curves for the first five days of SMA observations. These were the only tracks long enough for binning in time.

By $\Delta t=50$ days, the radio flux has diminished both due to the peak frequency shifting to lower frequencies, and to a decay in the peak flux. Specifically, the peak of the $15 \mathrm{GHz}$ light curve is $19 \mathrm{mJy}$ around 47 days (A. Horesh 2018, personal communication), substantially less luminous than the peak of the $230 \mathrm{GHz}$ or the $34 \mathrm{GHz}$ light curve. As we discuss in Section 4.2, this diminishing peak flux suggests that the interaction itself is diminishing, and enables us to constrain the size of the "circum-bubble" of material.

The X-ray light curve seems to have two distinct phases. We call the first phase ( $\Delta t \lesssim 20$ days) the plateau phase because the X-ray emission is relatively flat. The second phase, which we call the decline phase, begins around $\Delta t \approx 20$ days. During this period, the X-ray emission exhibits an overall steep decline, but also exhibits strong variation (by factors of up to 10) on shorter timescales (see also Kuin et al. 2018; Perley et al. 2018; Rivera Sandoval et al. 2018).
We use the shortest timescale of variability in the $230 \mathrm{GHz}$ light curve to infer the size of the radio-emitting region, and do the same for the X-ray emission in Section 5. On Days 5-6, the $230 \mathrm{GHz}$ flux changed by order unity in one day, setting a length scale for the source size of $\Delta R=c \Delta t=2.6 \times 10^{15} \mathrm{~cm}(170 \mathrm{au})$. We find no evidence for shorter-timescale variability in our long SMA tracks from the first few days of observations (Figure 2).

Together with the $230 \mathrm{GHz}$ flux density $\left(S_{\nu} \approx 30 \mathrm{mJy}\right)$ and the distance $(d=60 \mathrm{Mpc})$ we infer an angular size of $\theta=2.8 \mu$ as and a brightness temperature of

$$
T_{B}=\frac{S_{\nu} c^{2}}{2 k \nu^{2} \Delta \Omega} \gtrsim 3 \times 10^{10} \mathrm{~K}
$$

where $\Delta \Omega=\pi \theta^{2}$. This brightness temperature is close to the typical rest-frame equipartition brightness temperatures of the most compact radio sources, $T_{B} \sim 5 \times 10^{10} \mathrm{~K}$ (Readhead 1994). 

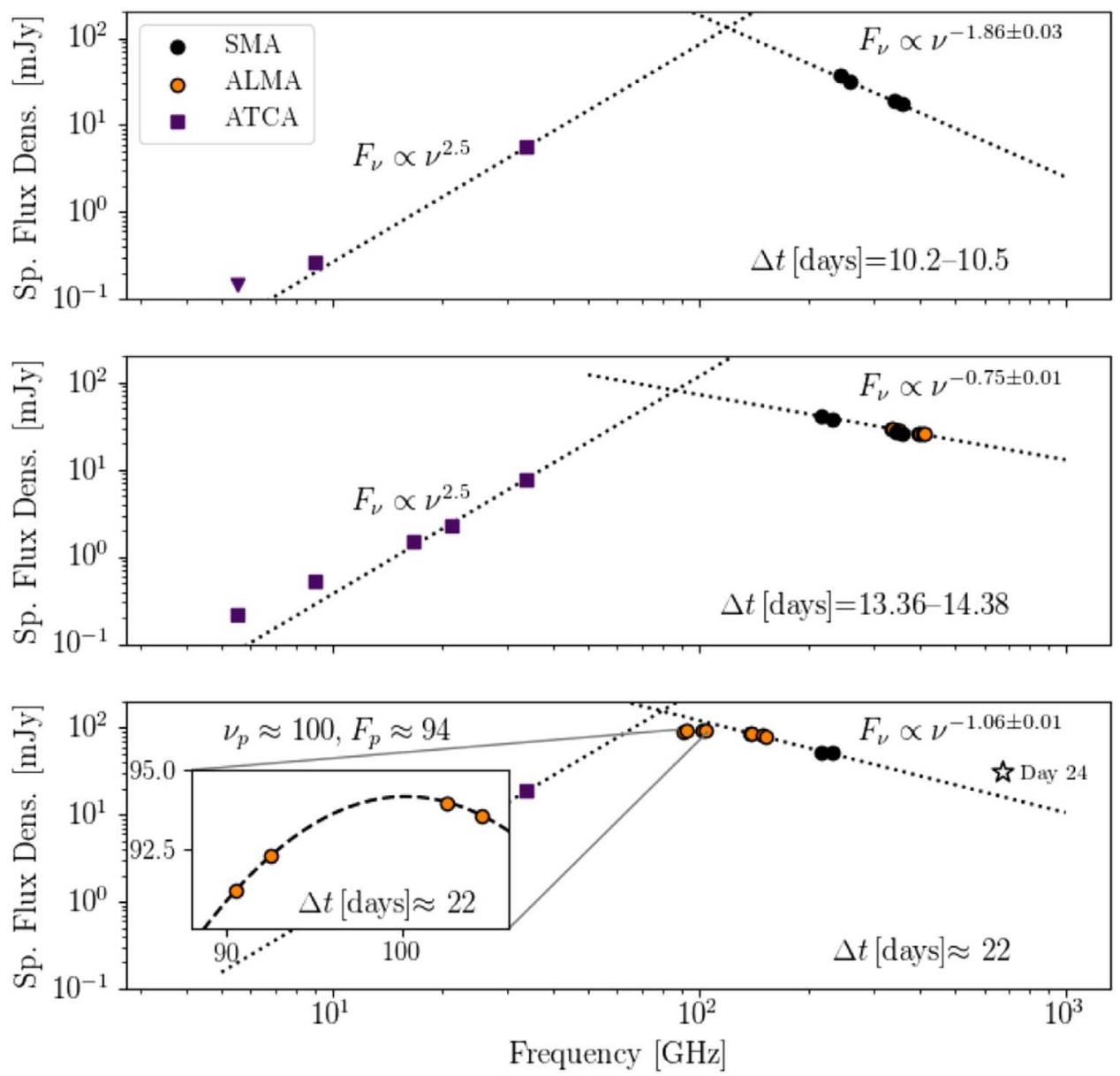

Figure 3. Spectrum of AT2018cow at three epochs. In the top panel, we plot the Day 10 data as presented in Table 1. In the middle panel, we plot the ATCA data from Day 13 and the SMA and ALMA data from Day 14. In the bottom panel, we plot the ALMA data from Day 22, interpolate the SMA data between Day 20 and Day 24 at 215.5 and $231.5 \mathrm{GHz}$, and interpolate the ATCA data at $34 \mathrm{GHz}$ (since it varies smoothly; Figure 1). We also show the Band 9 measurement from Day 24 as a star. The ATCA data is consistent with a self-absorbed spectral index $\left(F_{\nu} \propto \nu^{5 / 2}\right)$ with an excess at lower frequencies. The peak frequency is resolved on Day 22 with ALMA observations at Band 3 (see inset). To measure the optically thin spectral index, we performed a least squares fit in log space. To estimate the uncertainty on the spectral index, we performed a Monte Carlo analysis, sampling $10^{4}$ times to measure the standard deviation of the resulting spectral index. On Day 10, we used an uncertainty of 15\% for each SMA measurement. On Days 14 and 22, we used 10\% uncertainty for each ALMA measurement and 20\% for each SMA measurement (to take into account the much longer length of the SMA tracks). Uncertainties are too small to be visible on this plot, except for the inset panel, where we do not display them.

\subsection{Modeling the Radio to Submillimeter SED}

The shape of the radio to submillimeter SED (Figure 3), together with the high brightness temperature implied by the luminosity and variability timescale (Section 3.1), can only be explained by nonthermal emission (Readhead 1994). The observed spectrum is assumed to arise from a population of electrons with a power-law number distribution in Lorentz factor $\gamma_{e}$, with some minimum Lorentz factor $\gamma_{m}$ and electron energy power index $p$ :

$$
\frac{d N\left(\gamma_{e}\right)}{d \gamma_{e}} \propto \gamma_{e}^{-p}, \quad \gamma_{e} \geqslant \gamma_{m}
$$

As argued below, we expect an adiabatic strong shock moving into a weakly magnetized, ionized medium at a nonrelativistic speed. First-order Fermi acceleration gives $p=(r+2) /(r-1)$, where $r$ is the compression ratio of the shock. A strong matter-dominated shock has $r=4$, hence $p=2$ (Blandford \& Eichler 1987). However the back-reaction of the accelerated particles decelerates the gas flow, weakening the gas dynamic subshock and reducing the compression ratio from the strong shock $r=3$, so typical $2.5<p<3$ are obtained in both simulations and astrophysical data (Jones \& Ellison 1991). Quasi-perpendicular magnetized and relativistic shocks are more subtle, since some particles cannot return along field lines after their first shock crossing, but the limiting value is $p \sim 2.3$ (Pelletier et al. 2017).

Equation (3) provides an expression for $\gamma_{m}$. Behind the shock (velocity $v$ ) some fraction $\epsilon_{e}$ of the total energy density goes into accelerating electrons. Conserving shock energy flux gives

$$
\gamma_{m}-1 \approx \epsilon_{e} \frac{m_{p}}{m_{e}} \frac{v^{2}}{c^{2}} .
$$

The value of $\gamma_{m}$ is large for relativistic shocks, e.g., in GRBs. But we will see that for this source $\left(v / c \sim 0.1, \epsilon_{e} \sim 0.1\right)$, the bulk of the electrons are just mildly relativistic $\left(\gamma_{m} \sim 2-3\right)$. For ordinary SN shocks $\gamma_{m}$ is always nonrelativistic $\left(\gamma_{m}-1<1\right)$. Thus, in the parameter estimations below, we follow SN convention and assume that the relativistic electrons follow a power-law distribution down to a fixed $\gamma_{m}$ (Chevalier 1982, 1998; Kulkarni et al. 1998; Frail et al. 2000; Soderberg et al. 2005). We apply $\epsilon_{e}$ only to this relativistic power law, not to the nonrelativistic thermal distribution of shock-heated particles at lower energy. 
Table 3

Quantities Derived from Day 22 Measurements, Using Different Equipartition Assumptions

\begin{tabular}{lcc}
\hline \hline Parameter & $\epsilon_{e}=\epsilon_{B}=1 / 3$ & $\epsilon_{e}=0.1, \epsilon_{B}=0.01$ \\
\hline$\nu_{a}=\nu_{p}(\mathrm{GHz})$ & 100 & 100 \\
$F_{\nu, p}(\mathrm{mJy})$ & 94 & 94 \\
$r\left(10^{15} \mathrm{~cm}\right)$ & 7 & 6 \\
$v / c$ & 0.13 & 0.11 \\
$B(\mathrm{G})$ & 6 & 4 \\
$U\left(10^{48} \mathrm{erg}^{4}\right.$ & 4 & 35 \\
$n_{e}\left(10^{5} \mathrm{~cm}^{-3}\right)$ & 3 & 41 \\
$\nu_{c}(\mathrm{GHz})$ & 2 & 8 \\
\hline
\end{tabular}

Note. In the text unless otherwise stated we use $\epsilon_{e}=\epsilon_{B}=1 / 3$.

We now describe each of the break frequencies that characterize the observed spectrum. First, the characteristic synchrotron frequency $\nu_{m}$ emitted by the minimum energy electrons is:

$$
\nu_{m}=\gamma_{m}^{2} \nu_{g}
$$

where $\nu_{g}$ is the gyrofrequency,

$$
\nu_{g}=\frac{q_{e} B}{2 \pi m_{e} c}
$$

and $q_{e}$ is the unit charge, $B$ is the magnetic field strength, $m_{e}$ is the electron mass, and $c$ is the speed of light.

Next, there is the cooling frequency $\nu_{c} \equiv \nu\left(\gamma_{c}\right)$, the frequency below which electrons have lost the equivalent of their total energies to radiation via cooling. In general, the timescale for synchrotron cooling depends on the Lorentz factor as $t \propto \gamma_{e}^{-1}$. Thus, electrons radiating at higher frequencies cool more quickly. Separately, electrons could also lose energy by Compton upscattering of ambient (low energy) photons - the so-called Inverse Compton (IC) scattering. In Section 5, we find that IC scattering dominates at early times and that synchrotron losses dominate at later times, and that the transition is at $t \approx 13$ days.

At $\Delta t>13$ days, electrons with $\gamma_{e}>\gamma_{c}$ cool principally by synchrotron radiation to $\gamma_{c}$ in a time $t$, where

$$
\gamma_{c}=\frac{6 \pi m_{e} c}{\sigma_{T} B^{2} t}
$$

For $t<13$ days, Compton cooling on the UVOIR flux exceeds the synchrotron cooling rate by a factor $\sim(t / 10 \text { days })^{-5 / 2}$, and $\gamma_{c}$ is correspondingly lowered. The cooled electrons emit around the characteristic synchrotron frequency

$$
\nu_{c}=\gamma_{c}^{2} \nu_{g}
$$

Next, the self-absorption frequency $\nu_{a}$ is the frequency at which the optical depth to synchrotron self-absorption is unity. The rise at $34 \mathrm{GHz}$ obeys a $f_{\nu} \propto t^{2}$ power law (as shown in Figure 1), consistent with the optically thick spectral index we measure (Figure 3). This indicates that the self-absorption frequency is above the ATCA bands $\left(\nu_{a}>34 \mathrm{GHz}\right)$. Figure 3 also shows that the emission in the SMA bands is optically thin at $\Delta t \gtrsim 10$ days, constraining the self-absorption frequency to be $\nu_{a}<230 \mathrm{GHz}$.

On Day 22, we resolve the peak of the SED with our ALMA data. We denote the peak frequency $\nu_{p}$ and the flux at the peak frequency $F_{p}$, and find $\nu_{p} \approx 100 \mathrm{GHz}$ and $F_{p} \approx 94 \mathrm{mJy}$.
Motivated by the observation of optically thick emission at $\nu<\nu_{p}$, we assume that $\nu_{p}=\nu_{a}$, and adopt the framework in Chevalier (1998) (hereafter referred to as C98) to estimate properties of the shock at this epoch. These properties are summarized in Table 3, and outlined in detail below.

Following Equations (11) and (12) in C98, the outer shock radius $R_{p}$ can be estimated as

$$
R_{p}=\left[\frac{6 c_{6}^{p+5} F_{p}^{p+6} D^{2 p+12}}{\left(\epsilon_{e} / \epsilon_{B}\right) f(p-2) \pi^{p+5} c_{5}^{p+6} E_{l}^{p-2}}\right]^{1 /(2 p+13)}\left(\frac{\nu_{p}}{2 c_{1}}\right)^{-1}
$$

and the magnetic field can be estimated as

$$
B_{p}=\left[\frac{36 \pi^{3} c_{5}}{\left(\epsilon_{e} / \epsilon_{B}\right)^{2} f^{2}(p-2)^{2} c_{6}^{3} E_{l}^{2(p-2)} F_{p} D^{2}}\right]^{2 /(2 p+13)}\left(\frac{\nu_{p}}{2 c_{1}}\right)
$$

where, as in Equation (2), $p$ is the electron energy index. Note that C98 use $\gamma$ for the electron energy power index. We use $p$ instead and $\gamma$ for the Lorentz factor. The constant $c_{1}=6.27 \times 10^{18}$ in cgs units, and the constants $c_{5}$ and $c_{6}$ are tabulated as a function of $p$ on page 232 of Pacholczyk (1970). $D$ is the distance to the source, $E_{l}=0.51 \mathrm{MeV}$ is the electron rest mass energy, and $\epsilon_{e} / \epsilon_{B}$ is the ratio of energy density in electrons to energy density in magnetic fields (in C98 this ratio is parameterized as $\alpha$, but we use $\alpha$ as the optically thin spectral index of the radio SED.) Finally, $f$ is the filling factor: the emitting region is approximated as a planar region with thickness $s$ and area in the sky $\pi R^{2}$, and thus a volume $\pi R^{2} s$, which can be characterized as a spherical emitting volume $V=4 \pi f R^{3} / 3=\pi R^{2} s$.

On Day 22, we measure $\alpha=-1.1$ where $F_{\nu} \propto \nu^{\alpha}$, which corresponds to $p=3.2$. Later in this section we show that our submillimeter observations lie above the cooling frequency, and therefore that the index of the source function of electrons is $p_{s}=2.2$. However, the C98 prescription considers a distribution as it exists when the electrons are observed, from a combination of the initial acceleration and the energy losses (to cooling). So, we proceed with $p=3.2$, and discuss this unusual regime in Section 4.3. The closest value of $p$ in the table in Pacholczyk (1970) is $p=3$, so we use this value to select the constants (and note that, as stated in C98, the results do not depend strongly on the value of $p$.) With this, Equations (8) and (9) reduce to Equations (13) and (14) in $\mathrm{C} 98$, respectively, reproduced here:

$$
\begin{aligned}
& R_{p}=8.8 \times 10^{15}\left(\frac{\epsilon_{e}}{\epsilon_{B}}\right)^{-1 / 19}\left(\frac{f}{0.5}\right)^{-1 / 19} \\
& \times\left(\frac{F_{p}}{\mathrm{Jy}}\right)^{9 / 19}\left(\frac{D}{\mathrm{Mpc}}\right)^{18 / 19}\left(\frac{\nu_{p}}{5 \mathrm{GHz}}\right)^{-1} \mathrm{~cm}, \\
& B_{p}=0.58\left(\frac{\epsilon_{e}}{\epsilon_{B}}\right)^{-4 / 19}\left(\frac{f}{0.5}\right)^{-4 / 19} \\
& \times\left(\frac{F_{p}}{\mathrm{Jy}}\right)^{-2 / 19}\left(\frac{D}{\mathrm{Mpc}}\right)^{-4 / 19}\left(\frac{\nu_{p}}{5 \mathrm{GHz}}\right) \mathrm{G}
\end{aligned}
$$

Next we estimate the total energy $U$. For $p=3$, Equations (10) and (11) can be combined into the following 
expression for $U=U_{B} / \epsilon_{B}$,

$$
\begin{aligned}
U= & \frac{1}{\epsilon_{B}} \frac{4 \pi}{3} f R^{3}\left(\frac{B^{2}}{8 \pi}\right) \\
= & \left(1.9 \times 10^{46} \mathrm{erg}\right) \frac{1}{\epsilon_{B}}\left(\frac{\epsilon_{e}}{\epsilon_{B}}\right)^{-11 / 19}\left(\frac{f}{0.5}\right)^{8 / 19} \\
& \times\left(\frac{F_{p}}{\mathrm{Jy}}\right)^{23 / 19}\left(\frac{D}{\mathrm{Mpc}}\right)^{46 / 19}\left(\frac{\nu_{p}}{5 \mathrm{GHz}}\right)^{-1} .
\end{aligned}
$$

Following C98 we take $f=0.5$, but the dependence on this parameter is weak. In choosing $\epsilon_{B}$ and $\epsilon_{e}$ there are several normalizations (or assumptions) used in the literature. As a result the inferred energy can vary enormously (see Section 4.1 for further details). For now, we follow Soderberg et al. (2010) in setting $\epsilon_{e}=\epsilon_{B}=1 / 3$ (in other words, that energy is equally partitioned between electrons, protons, and magnetic fields). With all of these choices, we find that at $\Delta t \approx 22$ days, $R_{p} \approx 7 \times 10^{15} \mathrm{~cm}$ and $B_{p} \approx 6 \mathrm{G}$. We find that the total energy $U \approx 4 \times 10^{48} \mathrm{erg}$. Assuming $10 \%$ uncertainties in $F_{p}$ and $\nu_{p}$ and a $50 \%$ uncertainty in $p$, a Monte Carlo with 10,000 samples gives uncertainties of $0.15-0.3 \mathrm{dex}$ in these derived parameters. Our results are robust to departures from equipartition given the large penalty in the required energy (Readhead 1994).

The mean velocity up to $\Delta t \approx 22$ days is $v=R_{p} / t_{p}=$ $0.13 c$. We can write a general expression for $v / c$ (taking $L_{p}=4 \pi F_{p} D^{2}$, noting that $4 \pi \mathrm{Jy} \mathrm{Mpc}^{2}=1.2 \times$ $\left.10^{27} \mathrm{erg} \mathrm{s}^{-1} \mathrm{~Hz}^{-1}\right)$ :

$$
\begin{aligned}
& v / c \approx\left(\frac{\epsilon_{e}}{\epsilon_{B}}\right)^{-1 / 19}\left(\frac{f}{0.5}\right)^{-1 / 19} \\
& \times\left(\frac{L_{p}}{10^{26} \mathrm{erg} \mathrm{s}^{-1} \mathrm{~Hz}^{-1}}\right)^{9 / 19}\left(\frac{\nu_{p}}{5 \mathrm{GHz}}\right)^{-1}\left(\frac{t_{p}}{1 \text { days }}\right)^{-1} .
\end{aligned}
$$

Furthermore, from the $t^{2}$ rise at $34 \mathrm{GHz}$ (Figure 1) we can infer that the radius increases as $R \propto t$ and therefore that the velocity $v=d R / d t$ is constant. As shown in Figure 5, this is consistent with later-time, low-frequency observations by Margutti et al. (2018b). We put this derived energy and velocity into the context of other energetic transients in Section 4.1.

Next, we estimate the density of the medium into which the forward shock is propagating. The ejecta expands into the medium with velocity $v_{1}$, producing a shock front (a discontinuity in pressure, density, and temperature) with shock-heated ejecta immediately behind this front. Conservation of momentum across this (forward) shock front requires that

$$
P_{1}+\rho_{1} v_{1}^{2}=P_{2}+\rho_{2} v_{2}^{2}
$$

where $P$ is pressure (not to be confused with $p$ used as the power-law index for the electron energy distribution). The subscript 1 refers to the upstream (ambient) medium and the subscript 2 refers to the downstream medium (the shocked ejecta). Far upstream, the pressure can be taken to be 0 , and in the limit of strong shocks (for a monatomic gas) $\rho_{2} / \rho_{1}=$ $v_{1} / v_{2}=4$. Thus, this can be simplified to

$$
\frac{3 \rho_{1} v_{1}^{2}}{4}=P_{2}
$$

If the medium is composed of fully ionized hydrogen, $\mu_{p}=1$ and the number densities of protons and electrons are equal $\left(n_{p}=n_{e}\right)$. Using Equation (15) together with Equation (11), as well as the relations $P_{2}=\left(1 / \epsilon_{B}\right) B^{2} / 8 \pi$ and $\rho_{1}=\mu_{p} m_{p} n_{e}$,

$$
\begin{aligned}
& n_{e} \approx\left(20 \mathrm{~cm}^{-3}\right)\left(\frac{1}{\epsilon_{B}}\right)\left(\frac{\epsilon_{e}}{\epsilon_{B}}\right)^{-6 / 19}\left(\frac{f}{0.5}\right)^{-6 / 19} \\
& \times\left(\frac{L_{p}}{10^{26} \mathrm{erg} \mathrm{s}^{-1} \mathrm{~Hz}^{-1}}\right)^{-22 / 19}\left(\frac{\nu_{p}}{5 \mathrm{GHz}}\right)^{4}\left(\frac{t_{p}}{1 \text { days }}\right)^{2} .
\end{aligned}
$$

We find that the number density of electrons at $\Delta t \approx 22$ days is $n_{e} \approx 3 \times 10^{5} \mathrm{~cm}^{-3}$. We note that the strong jump conditions used here assume $\gamma=5 / 3$, and that there is a correction for the contribution of a relativistic $(\gamma=4 / 3)$ component. Chevalier (1983) quantified this correction using the factor $w$, the ratio of the relativistic pressure to the total pressure. In the most extreme case $(w=1)$ the correction is small, only a factor of 1.14 in $n_{e}$. This is negligible compared to our uncertainties.

At such a high density, the optical depth to free-free absorption (FFA) $\tau_{\text {ff }}$ might be expected to have a significant effect on the shape of the spectrum at low radio frequencies (Lundqvist \& Fransson 1988). From Lang (1999), we have

$$
\tau_{\mathrm{ff}}=8.235 \times 10^{-2}\left(\frac{T_{e}}{\mathrm{~K}}\right)^{-1.35}\left(\frac{\nu}{\mathrm{GHz}}\right)^{-2.1} \int\left(\frac{N_{e}}{\mathrm{~cm}^{-3}}\right)^{2}\left(\frac{d l}{\mathrm{pc}}\right)
$$

which, with our measured values of $n_{e}$ and $R$ on Day 22, gives the characteristic value

$$
\tilde{\tau}_{\mathrm{ff}}=68\left(\frac{T_{e}}{8000 \mathrm{~K}}\right)^{-1.35}\left(\frac{\nu}{\mathrm{GHz}}\right)^{-2.1}
$$

However, in AT2018cow the gas through which the shock is propagating is not at normal $\mathrm{H}$ II-region temperatures of $\sim 10^{4} \mathrm{~K}$. The UV and X-ray photons emitted at early times will completely ionize and Compton heat any surrounding gas: for gas at the density and radius given in Table 3, the lifetime to photoionization of a neutral hydrogen atom is less than $0.01 \mathrm{~s}$, while the recombination time is years. ${ }^{15}$ Compton heating of the electrons increases their temperature at the rate

$$
\frac{d(3 / 2) k T_{e}}{d t}=H=\frac{\sigma_{T}}{m_{e} c^{2}} \int_{0}^{\infty} \frac{h \nu L_{\nu} f_{\mathrm{KN}}\left(h \nu / m_{e} c^{2}\right)}{4 \pi R^{2}} d \nu
$$

where the Klein-Nishina correction $f_{\mathrm{KN}}(x) \simeq 1-21 x / 5+$ $O\left(x^{2}\right) \cdot{ }^{16}$ Even though the blackbody $(T=30,000 \mathrm{~K})$ luminosity at $\Delta t=3$ is 100 times larger than the coeval X-ray luminosity (Perley et al. 2018), the Compton heating is dominated by the 10-100 keV X-ray flux, and we find, for $3<\Delta t<20$ days, gas at the density and radius given in Table 3 has

$$
T_{e}(t) \simeq 1.0 \times 10^{6} \mathrm{~K}(t / 3 \text { days })^{0.6}
$$

\footnotetext{
${ }^{15}$ For much lower temperatures $T \sim 10^{4} \mathrm{~K}$, the Case B (high-density limit) recombination coefficient is $\alpha_{B}\left(T=10^{4} \mathrm{~K}\right)=2.6 \times 10^{-13} \mathrm{~cm}^{3} \mathrm{~s}^{-1}$ (Draine 2011), and the timescale is $t_{\text {recomb }}=1 /\left(\alpha_{B} n_{e}\right)$. For $n_{e}=3 \times 10^{5} \mathrm{~cm}^{-3}$, $t_{\text {recomb }} \approx 250$ days. This timescale becomes even longer for the expected higher temperatures.

16 Expressions for $f_{\mathrm{KN}}$ for cold electrons are given, e.g., in Equation (A1) of Sazonov et al. (2004) and Equation (5) of Madau \& Efstathiou (1999), and for finite temperature electrons in Equation (14) of Guilbert (1986).
} 
Given the spectral evolution shown in Perley et al. (2018), the Compton temperature (at which Compton heating balances Compton cooling) is $T_{c} \sim 2.5 \times 10^{6} \mathrm{~K}$ on Day 3 , hardening to $T_{c} \sim 1.8 \times 10^{7} \mathrm{~K}$ on Day 20 since the blackbody UV flux drops as $t^{-2.5}$, while the hard X-ray flux drops much more slowly. At these high Compton-heated temperatures $T_{e} \sim 10^{6} \mathrm{~K}$, the FFA optical depth given by Equation (18) only rises above unity below frequencies of $300 \mathrm{MHz}$, accessible to facilities like Low-Frequency Array (LOFAR).

Next, we estimate the luminosity from free-free emission of the ionized gas (Lang 1999):

$$
L \approx 1.43 \times 10^{-27} n_{e} n_{i} T^{1 / 2} V Z^{2} g \operatorname{erg~s}^{-1}
$$

where $n_{i}$ is the number density of ions, $Z$ is the atomic number, and $g \approx 1$ is the Gaunt factor, a quantum mechanical correction. Assuming that the gas is completely ionized out to the light-travel sphere at 22 days $\left(R=6 \times 10^{16} \mathrm{~cm}\right)$, we have $n_{e}=n_{i}$ in the region of interest. We also take $Z=1$. With the inferred density (Table 3 ) we find $L \approx 9 \times 10^{37} \mathrm{erg} \mathrm{s}^{-1}$, so the contribution to the observed X-ray luminosity is negligible.

Finally, we estimate the different break frequencies, beginning with $\nu_{m}$. Using Equation (3), taking $\epsilon_{e}=1 / 3$ and using our inferred $\beta=v / c$ from Day $22, \gamma_{m} \approx 5$. Next, using Equation (5) and our measured value of $B, \nu_{g} \approx 17 \mathrm{MHz}$. Equation (4) thus gives $\nu_{m} \approx 0.4 \mathrm{GHz}$, substantially below our peak frequency. The spectral index at $\nu_{m}<\nu<\nu_{a}$ is $\nu^{5 / 2}$ (Rybicki \& Lightman 1986), which we show as dotted lines in Figure 3. Clearly, the lowest-frequency fluxes are in excess of $\nu^{5 / 2}$ extrapolation. This naturally occurs if the source is inhomogeneous (e.g., magnetic field and/or particle energy density decreasing outwards). It can also arise even for a perfectly homogeneous source because the energy spectrum of the radiating electrons is not a pure power law: note that $\nu_{a}>\nu_{c}>\nu_{m}$, so even beyond the Maxwellian-like peak at $\gamma_{m}$, the spectrum is convex, steepening with energy above $\gamma_{c}$. These both produce self-absorbed spectra flatter than $\nu^{5 / 2}$ (see Section 6.8 in Rybicki \& Lightman (1986) and de Kool et al. (1989) for model calculations).

The cooling frequency due to synchrotron radiation is determined by Equations (6) and (7). We find $\gamma_{c} \approx 11$, giving a cooling frequency $\nu_{c} \approx 2 \mathrm{GHz}$. The relative contributions to electron cooling from synchrotron radiation and IC scattering are determined by the ratio between the radiation energy density and the magnetic energy density. On Day 22, the bolometric luminosity as measured in the UVOIR is $5 \times 10^{42} \mathrm{erg} \mathrm{s}^{-1}$ (Perley et al. 2018), so the radiation density is $u_{\mathrm{ph}}=0.26 \mathrm{erg} \mathrm{cm}^{-3}$. The magnetic energy density on the same day is $u_{B}=B^{2} / 8 \pi \approx 1.5 \mathrm{erg} \mathrm{cm}^{-3}$. Thus, synchrotron radiation is the dominant cooling mechanism, with a roughly $10 \%$ contribution from Inverse Compton (IC) scattering.

At this epoch, the cooling timescale $t_{\text {cool }}=\left(\gamma_{e} m_{e} c^{2}\right) /$ $\left(\frac{4}{3} \sigma_{T} u_{B} \gamma_{e}^{2} c\right)=240$ days $/ \gamma_{e}$ for an electron with $\gamma_{e}$, which is roughly 80 for an electron radiating at $100 \mathrm{GHz}$. So on Day 22 the cooling timescale is shorter than the timescale on which we are observing the source. This means that continuous reacceleration of the electrons is required, which could be provided by ongoing shock interaction.

As stated in Section 3.1, it seems that $\nu_{p}$ during the rise phase ( $\Delta t \approx 5-8$ days) was above or within the SMA observing bands. Using the peak observed flux and frequency as a lower limit on the peak flux and peak frequency, respectively, we consistently find that $v \approx 0.1 c$, albeit with a decreasing $n_{e}$ $\left(3 \times 10^{6} \mathrm{~cm}^{-3}\right.$ at $\left.\Delta t \approx 5\right)$.

\section{Implications of Shock Properties}

\subsection{AT2018cow in Velocity-energy Space, and a Discussion of Epsilons}

It is challenging to directly compare the energy of AT2018cow to that of other classes of radio-luminous transients, because there are several conventions that produce discrepant results. In particular, the energy partition fractions $\epsilon_{B}$ and $\epsilon_{e}$ are important for determining the total amount of energy in the shock, but are difficult to measure.

In the classical GRB literature, $\epsilon_{e}$ has been consistently measured to be $\epsilon_{e} \approx 0.2$, within a factor of 2 , while values of $\epsilon_{B}$ have much wider spreads, with a median value of $3 \times 10^{-5}$ but a distribution spanning four orders of magnitude (Kumar \& Zhang 2015). Kumar \& Barniol Duran (2010) constrain $\epsilon_{B} \sim 10^{-6}$ for a CSM density of $0.1 \mathrm{~cm}^{-3}$, and an even smaller value for higher densities. One of the best-observed GRB afterglows is GRB 130427A, and from modeling the evolving spectrum Perley et al. (2014) find $0.03<\epsilon_{B}<1 / 3$ and $0.14<\epsilon_{e}<1 / 3$.

There are different approaches to modeling for the handful of low-luminosity GRBs (LLGRBs) discovered to date. For LLGRB 980425/SN 1998bw, Kulkarni et al. (1998) invoked equipartition $\left(\epsilon_{B}=\epsilon_{e}=0.5\right)$. For LLGRB 031203/ SN2003 lw, Soderberg et al. (2004) used models from Sari et al. (1998) and Granot \& Sari (2002) together with the cooling frequency inferred from X-ray observations to estimate $\epsilon_{e}=0.4$ and $\epsilon_{B}=0.2$. For LLGRB 060218/SN2006aj, Soderberg et al. (2006) used the same prescription as was used in SN 1998bw. Finally, for LLGRB 100316D/SN2010bh, Margutti et al. (2013) set $\epsilon_{B}=0.01$ and allowed $\epsilon_{e}$ to vary from 0.01-0.1.

For Type II and Type Ibc radio $\mathrm{SNe}$, approaches range from using the $\mathrm{SN} 1998 \mathrm{bw}$ convention (i.e., $\epsilon_{B}=\epsilon_{e}=0.5$; Soderberg et al. 2005; Horesh et al. 2013) to $\epsilon_{B}=\epsilon_{e}=0.1$ (e.g., Chevalier \& Fransson 2006; Soderberg et al. 2006; Salas et al. 2013) to $\epsilon_{B}=\epsilon_{e}=1 / 3$ for the relativistic supernova SN 2009bb (Soderberg et al. 2010).

In this work, we follow the convention in Soderberg et al. (2010) so that we can compare our velocity-energy diagram to the corresponding diagram (Figure 4) in that paper. To put all transients on the same scale, we take the peak frequency, peak luminosity, and peak time for each event, and run them through the same equations that we used to infer the shock properties of AT2018cow. Note that we do not vary the values of $c_{5}, c_{6}, p$, but these are all very small corrections, whereas the effect of $\epsilon_{B}$ and the ratio $\epsilon_{e} / \epsilon_{B}$ in estimating the energy is large. The details of how we selected the peak values for each event are in Appendix C. When possible, we use the peak of the SED at a particular epoch. However, for most events, we use the peak flux density corresponding to a certain frequency, because well-sampled SEDs are rare.

Our rederived velocity-energy diagram is shown in Figure 4. AT2018cow has an energy comparable to mildly relativistic (LLGRBs; e.g., SN 1998bw) outflows and energetic SNe (e.g., SN 2007bg). We display vertical axes for two different conventions $\left(\epsilon_{B}=1 / 3\right.$ and $\left.\epsilon_{B}=0.01\right)$ to show how this 


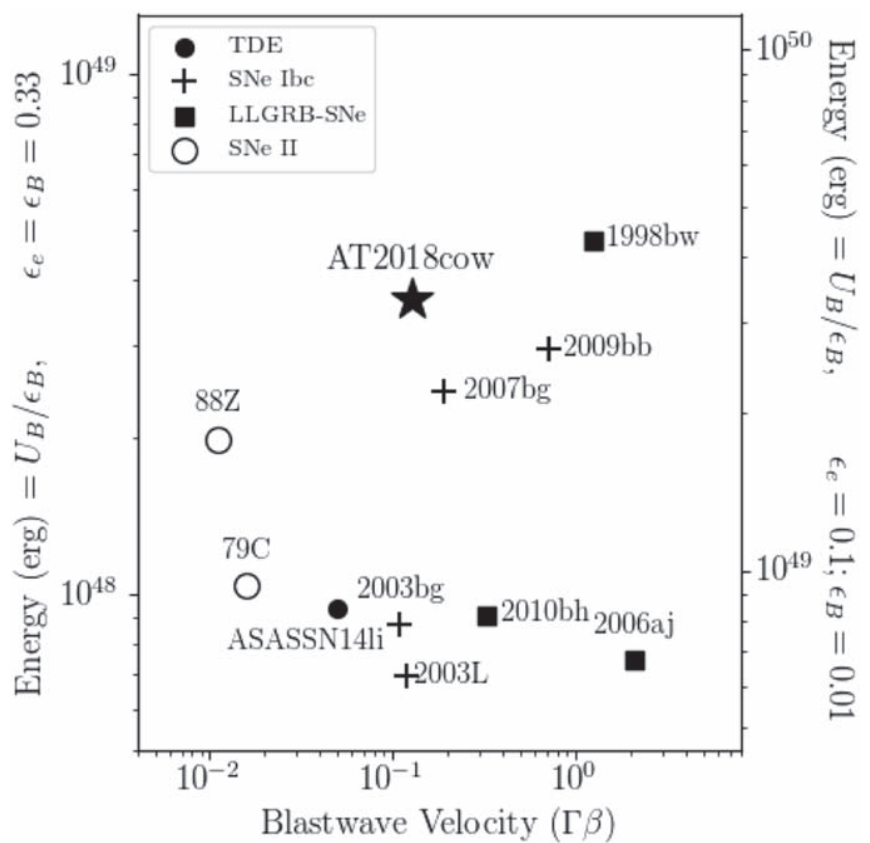

Figure 4. AT2018cow in velocity-energy space, compared to other classes of radio-luminous transients: TDEs (filled circles), Ibc SNe (crosses), $\mathrm{SNe}$ associated with LLGRBs (filled squares), and $\mathrm{SNe}$ II (open circles). For reference, GRBs lie above the plot at $10^{50} \mathrm{erg}<U<10^{52} \mathrm{erg}$, and the relativistic TDE Swift J1644 lies at $\approx 10^{51} \mathrm{erg}$ in this framework. For all sources, we take values of peak frequency and peak luminosity at some time (described in detail in Appendix C) and estimate velocity and energy using the same prescription that we use for AT2018cow. Estimates of energy are sensitive to the choice of $\epsilon_{B}$, as illustrated with the secondary axis on the right.

affects the inferred energy. Note that these values are not evaluated for a consistent epoch. However, for AT2018cow, we have reason to believe that the values of velocity and energy do not change significantly over the course of our observations. For other sources, it would be necessary to have a well-sampled SED over multiple epochs in order to trace the evolution of these values, and this is rare in the literature.

\subsection{A Luminous Millimeter Transient in a Dense Environment}

Here we compare the radio luminosity of AT2018cow to that of other transients, as observed at the spectral peak frequency at time $\Delta t$. As shown in Figure 5, AT2018cow stands out as being several times more luminous than SN 1998bw, and having a late peak at high frequencies. Over time, the peak luminosity diminishes to the value reported in the lowfrequency radio observations of Margutti et al. (2018b), supporting our inference that the velocity is not changing significantly.

On this diagram we also indicate lines of constant velocity (see Figure 3 of Soderberg et al. 2010 and Figure 4 of C98) and lines of constant mass-loss rate scaled by velocity $\dot{M} / v_{w}$, as a diagnostic of density (see Figure 10 of Jencson et al. 2018). Note that these lines assume $\nu_{p}=\nu_{a}$.

We now derive relations between the observational coordinates of the diagram in Figure 4 and physical quantities: the ordinate, peak radio luminosity $L_{p}$, is simply a power of the energy per unit radius $U / R$. We get an expression for $U / R$ using Equations (12) and (10):

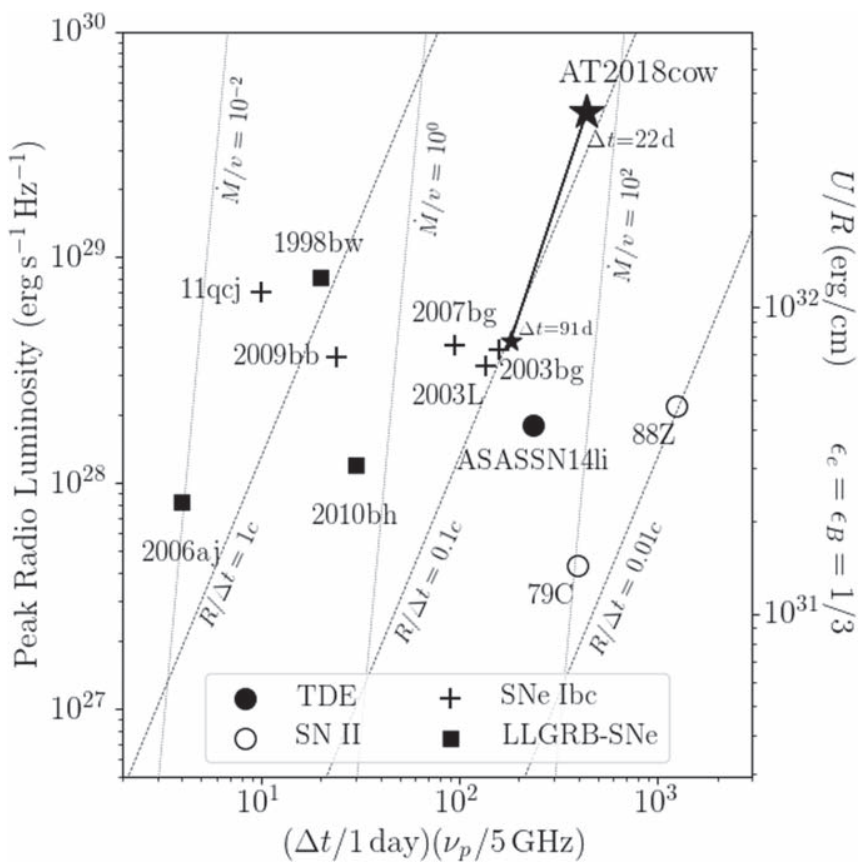

Figure 5. The peak luminosity of AT2018cow on two different epochs, compared to classes of energetic transients (see Chevalier 1998; Soderberg et al. 2010). The value at $\Delta t=22$ days comes from our work. The value at $\Delta t=91$ days comes from Margutti et al. (2018b) and shows that the velocity has not slowed significantly, which is consistent with the constant-velocity evolution we infer in Section 3.2 on the basis of early-time $34 \mathrm{GHz}$ data. For other sources, we choose values of peak frequency and peak luminosity as described in Appendix C. AT2018cow is unusual in having a large radio luminosity as well as a high $\nu_{a}$, and we discuss the physical interpretation of both of these characteristics in the text. Lines of constant mass-loss rate (scaled to wind velocity) are shown in units of $10^{-4} M_{\odot} \mathrm{yr}^{-1} / 1000 \mathrm{~km} \mathrm{~s}^{-1}$. Note that the dotted lines assume that the radio peak is due to synchrotron self-absorption rather than FFA, but that FFA has been the preferred fit in some cases, such as for SN 1979C and SN 1980K (Chevalier 1984).

$$
\begin{aligned}
& \frac{U}{R}=\left(3 \times 10^{29} \mathrm{erg} \mathrm{cm}^{-1}\right)\left(\frac{1}{\epsilon_{B}}\right) \\
& \times\left(\frac{\epsilon_{e}}{\epsilon_{B}}\right)^{-10 / 19}\left(\frac{f}{0.5}\right)^{9 / 19}\left(\frac{L_{p}}{10^{26} \mathrm{erg} \mathrm{s}^{-1} \mathrm{~Hz}^{-1}}\right)^{14 / 19} .
\end{aligned}
$$

This translation between $L_{p}$ and $U / R$ is shown on the left and right axis labels of Figure 4.

We now show that the abscissa of Figure 5, ( $\Delta t / 1$ days $)\left(\nu_{p} / 5 \mathrm{GHz}\right)$, is very nearly proportional to the square root of the swept up mass per unit radius $M / R$, or equivalently, if the surrounding medium was from a pre-explosion steady wind of speed $v_{w}, \dot{M} / v_{w} \propto M / R$. A steady spherical wind of ionized hydrogen with velocity $v_{w}$ has $n_{e}=\dot{M} /\left(4 \pi m_{p} r^{2} v_{w}\right)$, so we can reparameterize the density in terms of the mass-loss rate:

$$
\begin{aligned}
\frac{\dot{M}}{v_{w}}\left(\frac{1000 \mathrm{~km} \mathrm{~s}^{-1}}{10^{-4} M_{\odot} \mathrm{yr}^{-1}}\right)= & (0.0005)\left(\frac{1}{\epsilon_{B}}\right)\left(\frac{\epsilon_{e}}{\epsilon_{B}}\right)^{-8 / 19} \\
& \times\left(\frac{f}{0.5}\right)^{-1 / 19}\left(\frac{L_{p}}{10^{26} \mathrm{erg} \mathrm{s}^{-1} \mathrm{~Hz}^{-1}}\right)^{-4 / 19} \\
& \times\left(\frac{\nu_{p}}{5 \mathrm{GHz}}\right)^{2}\left(\frac{t_{p}}{1 \text { days }}\right)^{2} .
\end{aligned}
$$


Notice the weak $\left(-4 / 19\right.$ power) dependence on $L_{p}$, and the quadratic dependence on $\nu_{p} t_{p}$, which means the lines of constant $\dot{M} / v_{w}$ are nearly vertical in Figure 5 .

AT2018cow lies along the same velocity line as SN 2003bg and SN 2003L, but $n_{e}$ is a factor of a few to an order of magnitude larger. ${ }^{17}$ Similarly, SN 1998bw lies along a similar velocity line to $\mathrm{SN} 2006 \mathrm{aj}$, but $n_{e}$ (using our prescription) is $40 \mathrm{~cm}^{-3}$, while the density inferred for 2006aj using the same prescription is $3 \mathrm{~cm}^{-3}$. For $\mathrm{SNe} \mathrm{Ibc}$ in general, Chevalier \& Fransson (2006) attributed the large spread in radio luminosity to a spread in circumstellar density, with the example that SN 2002ap (not shown in Figure 5 due to its relatively low luminosity) is roughly three orders of magnitude less luminous than SN 2003L, and its inferred ambient density is also a factor of 3 smaller. In SN 2003L and SN 2003bg, the high density was attributed to a stellar wind.

This is not the whole story: as we showed above, high peak radio luminosity just corresponds to high $U / R$, i.e., high energy and/or small radius. Since $U$ is the converted energy, it represents only a lower limit to the actual driving kinetic energy (becoming equal to it as the explosion transitions from free expansion to the Sedov phase). A higher-density medium more quickly converts the piston's energy to thermal energy than does a low-density medium. Thus, for a large fixed explosion energy, a denser medium will indeed lead to larger peak radio luminosities. But the direct correlation is with the (thermalized) energy per unit radius, $U / R$. Similarly, Equation (11) shows that (except for a very weak $L_{p}^{-2 / 19}$ dependence), $\nu_{p} \propto B_{p}$. Thus, higher peak frequencies are directly indicative of a higher magnetic field, or equivalently, pressures. Therefore, AT2018cow's high $\nu_{p}$ and high $L_{p}$ are quite likely mostly a consequence of it being energetic, and observed early, when the high wind density at small radii led to high pressure, and enhanced $U / R$. As we discuss below, this suggests that many other $\mathrm{SNe}$ could have shown similar bright mm-submm fluxes, had they been observed at those wavelengths in their first week.

On Day 22, the inferred density is $\rho_{0}=4 \times 10^{-19} \mathrm{~g} \mathrm{~cm}^{-3}$ at a radius of $r_{0}=7 \times 10^{15} \mathrm{~cm}$ (Table 3). From this we can infer $\dot{M} / v_{\mathrm{w}}=2.4 \times 10^{14} \mathrm{~g} \mathrm{~cm}^{-1}$. The mass swept up to radius $r$ is $\left(\dot{M} / v_{\mathrm{w}}\right) r$. In Section 3, we argue that the blast wave reaches the edge of the surrounding bubble around $\Delta t \approx 50$ days. If so, given our inferred velocity, the radius of the circum-bubble is $1.7 \times$ $10^{16} \mathrm{~cm}$, and the mass of the circum-bubble $\approx 2 \times 10^{-3} M_{\odot}$. The mass-loss rates for hot stars $\left(v_{w} \sim 2000 \mathrm{~km} \mathrm{~s}^{-1}\right.$, Lamers \& Leitherer 1993) and red supergiants $\left(v_{w} \sim 20 \mathrm{~km} \mathrm{~s}^{-1}\right.$, van Loon 2010) range from $10^{-4}-10^{-6} M_{\odot} \mathrm{yr}^{-1}$ (Smith et al. 2018). Thus, $r_{0} / v_{w}$ is $\sim 1 \mathrm{yr}$ for a hot star progenitor, and $\sim 100 \mathrm{yr}$ for a red supergiant. Therefore, the circum-bubble could either have been formed by normal mass loss in a red supergiant, or end-of-life enhanced mass loss from a hot star or red supergiant (see, e.g., Smith et al. 2017 and references therein).

UVOIR observations of AT2018cow place strong constraints on the nature of the surrounding medium (Perley et al. 2018). The high luminosity and fast rise can be interpreted as

\footnotetext{
17 The radial density profile inferred for SN 2003bg is $n_{e} \approx 2.2 \times$ $10^{5}\left(r / r_{0}\right)^{-2} \mathrm{~cm}^{-3}$ (Soderberg et al. 2006) and the radial density profile inferred for SN 2003L is $n_{e} \approx 6.1 \times 10^{4}\left(r / r_{0}\right)^{-2} \mathrm{~cm}^{-3}$ (Soderberg et al. 2005). In both cases, $r_{0} \approx 10^{15} \mathrm{~cm}$ is the shock radius at $t_{0}=10$ days. For SN 2003L, we infer $t_{0}$ using the result that $r=3 \times 10^{15} \mathrm{~cm}$ at $t=28$ days, and that $\alpha_{r}=0.96$ in the parameterization $r=r_{0}\left(t / t_{0}\right)^{\alpha_{r}}$ (Model 1 in Soderberg et al. 2006).
}

shock heating of a dense shell of material at $R=10^{14} \mathrm{~cm}$ or $10 \mathrm{au}$, qualitatively consistent with the inference of dense material given the properties inferred from the radio shock. On the other hand, early spectra show no narrow emission lines indicative of a shock and the light curve declines steeply after peak, both of which suggest that this dense material must also be quite limited in extent, with little material at larger radii. While the radio observations also suggest that a cutoff in the density distribution may exist, the $0.1 c$ shock does not reach it for almost 20 days, a quite different timescale than the optical peak (reached in less than 3 days) or early spectroscopy. This might be due to the $0.1 c$ shock being produced by breakout from the $R=10^{14} \mathrm{~cm}$ shell, which re-energized the (much slower) SN shock. Or there could be deviations from spherical symmetry (for example, with the optical heating a quasispherical shell but the radio shock passing through a denser toroidal component or clouds along a bipolar jet).

Thus, an energetic shock propagating into a dense environment could produce a radio SED that peaks at submillimeter wavelengths at early times. However, as illustrated in the left panel of Figure 6, searches at high frequencies at early times have been rare, and primarily limited to transients with relativistic jetted outflows (GRBs, TDEs). We suggest that these searches be expanded to other classes of transients: luminous $\mathrm{SNe}$ such as SN 2003L and SN 2007bg, and luminous TDEs such as ASASSN14li, all exploded into dense media and exhibited luminous centimeter-wavelength emission at $t>10$ days. As time goes on, the SED peak shifts to lower frequencies and diminishes in brightness, so these events could have been bright millimeter transients at $t<10$ days. This is supported by Figure 5, which shows that SN 2007bg, SN 2003bg, and SN 2003L could have appeared similar to AT2018cow had they been observed earlier at higher frequencies.

\subsection{Novel Features of the Synchrotron Model Parameters}

The ordering of the break frequencies, $\nu_{\mathrm{ff}}<\nu_{m}<\nu_{c}<\nu_{a}$, is an unusual regime for long-wavelength observations. For a relativistic shock (GRBs), the typical orderings are $\nu_{a}<\nu_{c}<\nu_{m}$ (the fast cooling regime) and $\nu_{a}<\nu_{m}<\nu_{c}$ (the slow cooling regime; Sari et al. 1998). For nonrelativistic shocks, the ordering in most cases seems to be $\nu_{a}<\nu_{m}<\nu_{c}$ at measured frequencies above $1.4 \mathrm{GHz}$, but can also be $\nu_{m}<\nu_{a}<\nu_{c} ; \nu_{c}$ is typically considered unimportant for longwavelength observations (Nakar \& Piran 2011).

The low cooling frequency is a consequence of a large magnetic field strength, $\nu_{c} \propto B^{-3}$ (reduced even further for $t<10$ days by Compton cooling on the UVOIR flux, which dominates over synchrotron cooling). This in turn presumably arises from the injection of a large amount of energy into a small volume of material, consistent with the low velocity we measure. From Equation (11) we see that $B_{p}$ scales as $\left(\epsilon_{e} / \epsilon_{B}\right)^{-4 / 19} L_{p}^{-2 / 19} \nu_{p}$. Changing $\epsilon_{B}$ from $1 / 3-0.01$ could increase $\nu_{c}$ by a factor of 8 , still much lower than our observed frequencies. This regime is selectively probed by submillimeter observations, because a low $\nu_{c}$ (high $B_{p}$ ) gives rise to a $\nu_{a}$ that falls in this wavelength regime. We note that in the same framework, the relativistic TDE Swift J1644+57 (whose longwavelength SED also peaked in the submillimeter for the first few weeks) would also have had a cooling frequency below much of the observed frequency range $\left(\nu_{c} \approx 6 \mathrm{GHz}\right)$.

Since $\nu_{c}$ is below any of our measured frequencies, the injection spectrum (the spectrum of the electrons prior to 

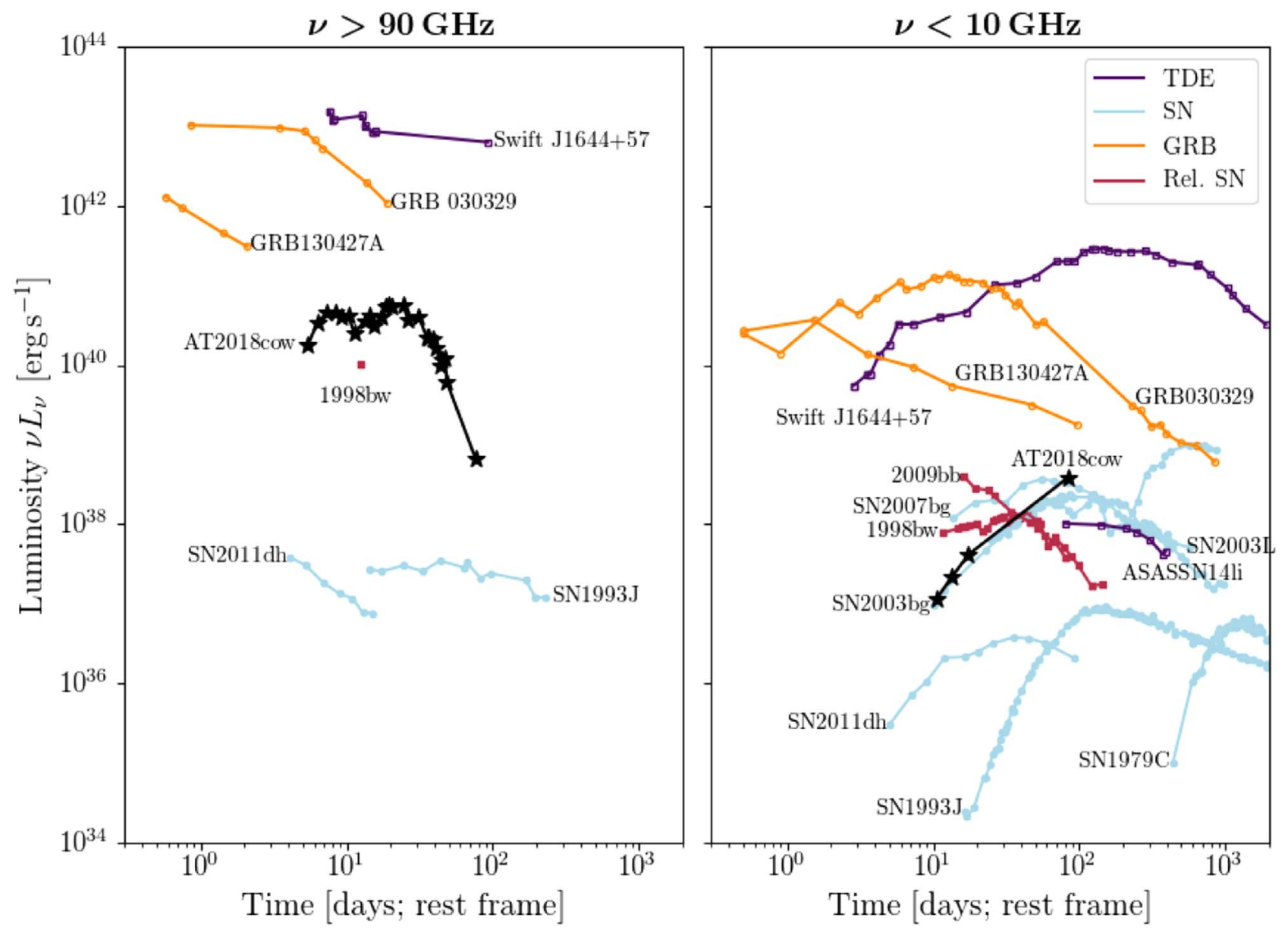

Figure 6. Luminosity evolution for different transients, measured at high frequencies $(\nu>90 \mathrm{GHz}$; left panel) and low frequencies $(\nu<10 \mathrm{GHz}$; right panel). Classes are GRBs (orange open circles; Berger et al. 2003; Sheth et al. 2003; Perley et al. 2014), TDEs (purple open squares; Zauderer et al. 2011, 2013; Berger et al. 2012; Alexander et al. 2016; Eftekhari et al. 2018), nonrelativistic SNe (light blue-filled circles; Weiler et al. 1986, 2007; Soderberg et al. 2005, 2006; Krauss et al. 2012; Horesh et al. 2013; Salas et al. 2013), and relativistic SNe (red-filled squares; Kulkarni et al. 1998; Soderberg et al. 2010). Thus, there are a number of transients measured with radio telescopes (relativistic SN 2009bb, energetic SNe 2003L, 2003bg, and 2007bg) that could have been bright millimeter transients but were not observed at high frequencies. The late-time low-frequency AT2018cow point is from Margutti et al. (2018b).

cooling) has a shallower power-law index than what we measure, $p_{i}=p-1$. This suggests that $p_{i} \approx 2.2$ on Day 22 , when $p \approx 3.2$, This is not unreasonable for Fermi acceleration from a strong shock. Typical young Galactic SN remnants have $p=p_{i}=2.4$ in the radio, flattening to $p \sim 2$ at higher frequencies (Urošević 2014).

\section{Origin of the X-Ray Emission and Emergence of a Compact Source}

During the plateau phase, the fluence in the Swift/XRT bands is $\int F_{X} d t \approx 1.7 \times 10^{-5} \mathrm{erg} \mathrm{cm}^{-2}$. Integrating the Swift/ XRT light curve until $\Delta t=22$ days, we find $7 \times 10^{48} \mathrm{erg}$. Using the NUSTAR spectra index $\alpha=0.5$, extrapolating this to $100 \mathrm{keV}$ would increase this energy by a factor of 3 .

The total X-ray energy emitted in the first 3 weeks is thus greater than the total energy in the shock inferred from radio observations on Day $22, U \approx 4 \times 10^{48} \mathrm{erg}$. If a significant proportion of the X-rays is produced by IC emission, then our assumption of $\epsilon_{B}=\epsilon_{e}=1 / 3$ clearly results in an underestimate of the total energy, and an assumption of $\epsilon_{B}=0.01$ would be more appropriate. As shown in Figure 4, $U$ would be increased by a factor of 9 with the assumption $\epsilon_{B}=0.01$ and $\epsilon_{e}=0.1$, just barely comparable to the total energy emitted in $\mathrm{X}$-rays.

The luminosity of the UVOIR source declines $\propto t^{-\beta}$, where $\beta \approx 2.5$ (Perley et al. 2018). Assuming a constant expansion speed for the shock of $0.13 c$ (see Table 3 and related discussion in Section 3) the photon energy density of the UVOIR source, $u_{\mathrm{ph}}=L_{\mathrm{UVOIR}}(T) /\left(c 4 \pi R(t)^{2}\right)=5.2(t / 10 \text { days })^{-9 / 2}\left(\epsilon_{e} /\right.$ $\left.\epsilon_{B}\right)^{2 / 19} \mathrm{erg} \mathrm{cm}^{-3}$. Assuming that the magnetic field pressure scales with the ram pressure of the shock $\left(\rho_{1} v_{1}^{2}\right)$ and assuming $\rho \propto r^{-2}$, from Equation (11), the magnetic energy density $u_{B}=7.4\left(t / 10\right.$ days $^{-2}\left(\epsilon_{B} / \epsilon_{e}\right)^{8 / 19} \mathrm{erg} \mathrm{cm}^{-3}$. For $\epsilon_{B}=0.01$, $\epsilon_{e}=0.1, u_{\mathrm{ph}} / u_{B}=2(t / 10 \text { days })^{-2.5}$, with only a rather weak dependence on the epsilons. This ratio is equal to unity around $t=13$ days, marking the transition from a regime dominated by Compton cooling to a regime dominated by synchrotron cooling.

This ratio is much lower than the observed ratio $L_{X} / L_{\text {radio }} \gtrsim 30$, and the X-ray spectral index is also substantially flatter than the radio spectral index. We conclude that the $\mathrm{X}$-ray emission during the plateau phase does not naturally arise from IC scattering of the UVOIR source by the electrons in the post-shocked region (which also generate the radio to submillimeter emission via synchrotron radiation): IC from the 


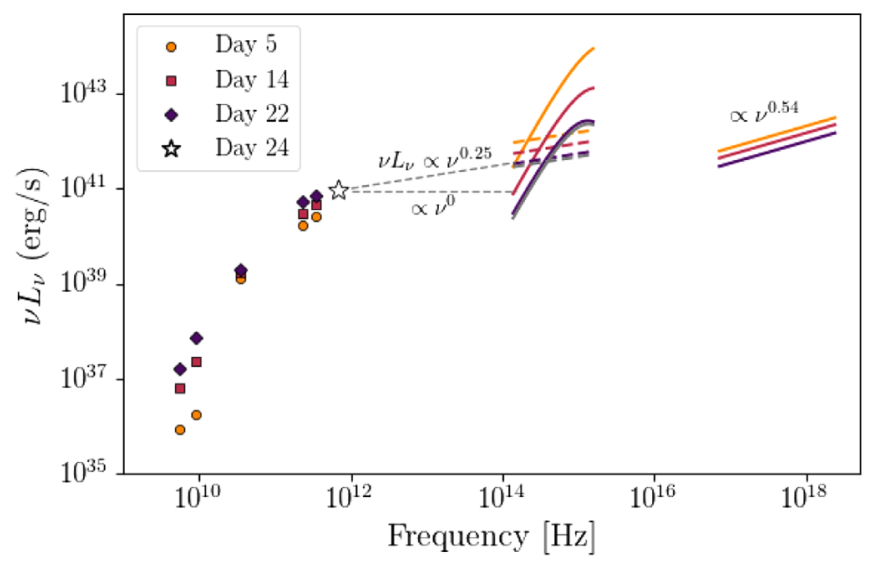

Figure 7. The full radio to X-ray SED. Since the ATCA data vary smoothly over the course of our observations, we fit a power law to the existing light curves (see Figure 1) and plot the values for the given day at 5.5, 9, and $34 \mathrm{GHz}$. For the SMA data, we interpolate the spectrum for the given day and plot the value at 231.5 and $345 \mathrm{GHz}$. We plot the ALMA data as measured, including the single Band 9 measurement (white star), which seems to show an excess above the other radio data. We plot the best-fit blackbody and nonthermal component from Perley et al. (2018), and show that the nonthermal component could be an extension of the excess seen in Band 9 on Day 24. We plot the Swift/XRT data as follows: we interpolate the light curves to estimate the integrated $0.3-10 \mathrm{keV}$ flux at the given epoch. We use the geometric mean of $(0.3 \mathrm{keV}, 10 \mathrm{keV})$ and the spectral index $\nu^{0.54}$ to solve for the normalization coefficient for the spectrum. We display the spectrum across the full XRT range.

radio-mm emitting region alone underpredicts the X-ray luminosity, predicts an X-ray luminosity declining much more rapidly than observed in the first 20 days, and predicts too steep a spectrum. It also does not naturally arise from an extension of the $\alpha \approx-1.1$ radio-submm synchrotron spectrum: the $\mathrm{X}$-ray emission is some 25 times brighter than that extrapolation (see Figure 7$)$, and has a much flatter $(\alpha \approx-0.5)$ spectral index. Further speculative modeling of the source of the X-ray emission during the plateau phase is beyond the scope of this paper.

During the decline phase $t>20$ days, the timescale of these fluctuations is around $0.05 t$, while the diameter we infer for the radio-emitting region (see Section 3) is $\sim 2 \times 0.13 t=0.26 t$. Thus, the X-ray emission must arise in a different and more compact source than the radio-emitting shell.

From the plateau phase to the decline phase, the X-ray emission softens, as shown in the bottom panel of Figure 1 and reported by Kuin et al. (2018). From the NuSTAR data, measuring the flux using cflux, we infer a hardness ratio $L_{X}(10-200 \mathrm{keV}) / L_{X}(0.3-10 \mathrm{keV}) \approx 26$ on Epoch 1 , similar to what is inferred by Kuin et al. (2018) using a joint BAT/XRT analysis. From the $N U S T A R$ data, we find a hardness ratio of $L_{X}(10-200 \mathrm{keV}) / L_{X}(0.3-10 \mathrm{keV}) \approx 4-5$ on Epochs 3 and 4 . This is consistent with other studies, which found negligible spectral evolution in the Swift $0.3-10 \mathrm{keV}$ band, but significant spectral evolution at higher energies (Kuin et al. 2018; Margutti et al. 2018b; Rivera Sandoval et al. 2018).

These two changes - the onset of variability five times faster than the light-travel time across the radio-emitting shell, and the striking change in the spectrum-lead us to conclude that beyond 20 days the X-ray emission arises from a different and more compact source than during the plateau phase. In the decline phase we are, arguably, probing regions closer to the central engine of the event.
The peculiarities of the UVOIR spectrum have led some to propose that AT2018cow is a TDE of a white dwarf by an $\sim 10^{5-6} M_{\odot}$ black hole (Kuin et al. 2018; Perley et al. 2018). Given its off-nucleus location (1.7 kpc; Perley et al. 2018) in a star-forming galaxy, and the similarities of the radio-emitting shock to those of other $\mathrm{SNe}$, it seems more natural to suppose that AT2018cow originated in a stellar cataclysm. Ultimately, however, our radio observations only require a $v \sim 0.1 c$ shock wave propagating into a dense medium, which could very plausibly arise in both TDE and SN models. The radio observations do little to distinguish them. In either picture, the striking late-time change in the X-ray behavior suggests the emergence of a central engine. In the TDE case, this could be an accretion disk around a black hole. In the stellar explosion case, this could be a natal black hole accreting (fallback) matter from the debris, or a magnetar. The emergence could then be due to a channel between the interior and the surface opened up by a collimated outflow (a "jet" or stifled jet's cocoon breakout; Nakar 2015), or to gaps in the photosphere opened by Rayleigh-Taylor instabilities.

We now briefly explore the magnetar model, which has been proposed for cosmological long-duration GRBs (e.g., Thompson et al. 2004) and superluminous SNe (e.g., Kasen \& Bildsten 2010). Prentice et al. (2018) invoked a magnetar model to explain the UVOIR observations of AT2018cow and found a best-fit magnetic field strength of $2 \times 10^{15} \mathrm{G}$ and a best-fit spin period of $11 \mathrm{~ms}$. Note that the magnetar itself need not be directly visible: the X-rays we see could be due to the emergence of bubbles of the magnetar-powered wind nebula (Kasen et al. 2016). The spin-down luminosity of a magnetar with period $P$ is $L \propto \omega \dot{\omega}$, where $\omega=2 \pi / P$ is the angular frequency. The spin-down timescale is $\tau_{c}=P / 2 \dot{P}$. We set $\tau_{c}=20$ days, $L_{X}=5 \times 10^{42} \mathrm{erg} \mathrm{s}^{-1}$ and find $P=50 \mathrm{~ms}$ and $\dot{P}=1.4 \times 10^{-8} \mathrm{~s} \mathrm{~s}^{-1}$, assuming that all the spin-down power goes into X-ray production. For a constant spin-down rate, this would correspond to an initial spin period of $26 \mathrm{~ms}$, similar to the result in Prentice et al. (2018) for the model fit to the griz light curve.

With $P$ and $\dot{P}$ in hand, using the standard dipole formula, we find a lower limit on the magnetic field strength of $8 \times 10^{14} \mathrm{G}$, which is consistent with the value found in Prentice et al. (2018). Our modeling of the forward shock led to a lower limit to the energy of $U \approx 10^{49} \mathrm{erg}$, depending on the value of $\epsilon_{B}$. If this was supplied by a magnetar, then the initial period of the magnetar is $\lesssim 10 \mathrm{~ms}\left(U / 10^{50} \mathrm{erg}\right)^{-1 / 2}$. We end this discussion by noting that the spin-down luminosity in the dipole model (with constant B-field) is $\propto t^{-2}$, which is roughly consistent with the slope of the decay of the X-ray light curve. This is, however, not easily distinguished from the $\propto t^{-5 / 3}$ slope expected from accretion in a TDE (Phinney 1989) or fallback (Michel 1988).

If this is a stellar explosion, then the features in the UVOIR spectra and the rise time point to an extended progenitor $\left(10^{14} \mathrm{~cm}\right.$; Perley et al. 2018), comparable in size to the largest red supergiants. This is not consistent with the compact, stripped stars invoked as progenitors for other classes of engine-driven explosions like GRBs and SLSNe (although see Smith et al. (2012) for a possible exception). As discussed in Perley et al. (2018) and Section 4.2, a more likely scenario is that the progenitor experienced a dramatic, abrupt episode of mass loss shortly before the explosion, and the UVOIR 
photosphere lies within this "brick wall," which the SN blast wave struck and re-thermalized.

Regardless of the nature of the central engine, we have the following model: the fastest-moving ejecta races ahead at $v_{1}=0.13 c$ into a dense circum-bubble of radius, $R_{b}$. In the post-shocked gas, electrons are accelerated into a power-law spectrum and magnetic fields are amplified. We attribute decay of the resulting radio emission at $t_{b}=50$ days to the fastmoving ejecta reaching the edge of this circum-bubble and infer a radius $R_{b}=v_{1} t_{b} \approx 1.7 \times 10^{16} \mathrm{~cm}$, and a mass of $10^{-3} M_{\odot}$. Within the radio-emitting shell is a long-lived engine, which may inflate a bubble of plasma and magnetic fields (Bucciantini et al. 2007). There is also slower ejecta heated by a central source (or radioactivity) that expands and emits UVOIR radiation. The photosphere of this component recedes with time, and at early times its large Compton optical depth obscures direct emission from the vicinity of the central engine. At later times, this central region emerges.

\section{Conclusions and Outlook}

Persuasive arguments can and have been made for both SN and TDE origins for AT2018cow. Our extensive radio through submillimeter observations enable us to draw definitive conclusions about the outer blast wave launched following the event, independent of the origin of the event. The blast wave is sub-relativistic $(v / c=0.13)$ and plows into a dense medium $\left(n_{e}=3 \times 10^{5} \mathrm{~cm}^{-3}\right.$ at $\left.R \sim 7 \times 10^{15} \mathrm{~cm}\right)$. The energy contained within this blast wave is $U \approx 10^{49 \pm 0.3} \mathrm{erg}$. In contrast to the UVOIR luminosity, which declines as $L_{\mathrm{UVOIR}} \propto t^{-2.5}$ over the period of 3 days $<t<20$ days, the $\mathrm{mm}$-submm and X-ray luminosities are both relatively constant over the same period (with $\sim 50 \%$ variations over timescales of a few days, comparable to the light-travel time across the radioemitting shell, $t_{l c}=2 v t / c=0.26 t$; see Figure 1 ).

The initially attractive idea of attributing the X-rays to IC scattering of the rapidly declining flux of UVOIR photons by relativistic electrons (which give rise to the radio and submillimeter flux) is not naturally consistent with the slow decline of the $\mathrm{X}$-ray and radio-submm flux, the X-ray luminosity, or the X-ray spectral index. Thus, we are forced to invoke an additional source of X-ray emission during both the $t<20$ days plateau phase, and during the decline phase after 20 days, when the X-rays begin to fade and show dramatic variations now on timescales several times shorter than the light-travel time across the radio-emitting shell. These are suggestive of power from a central engine, which could be consistent with either a stellar explosion or a TDE. Future X-ray monitoring may be useful in differentiating between central engine models; in particular, a power-law decay $\left(t^{-2}\right)$ is a distinct signature of the magnetar model, although difficult in practice to distinguish from the $t^{-5 / 3}$ expected from fallback or a TDE.

The radio source is remarkable even on purely observational grounds. The peak radio luminosity (nearly $10^{41} \mathrm{erg} \mathrm{s}^{-1}$ ) greatly exceeds that of the most radio-luminous $\mathrm{SNe}$ and "normal" TDEs, and is surpassed only by relativistic jetted transients (GRBs and TDEs). The source remains luminous at submillimeter wavelengths for nearly a month, with a selfabsorption frequency $\nu_{a} \sim 100 \mathrm{GHz}$ at $\Delta t \approx 22$ days.

The source is strongly detected at nearly a terahertz (ALMA Band 9; $671 \mathrm{GHz}$ ) even 3 weeks post discovery. We note that the Band 9 flux is higher than the extrapolation based from lower frequency bands (Figure 3, middle panel), and intriguingly connects to the NIR nonthermal component suggested by Perley et al. (2018). However, we readily admit that the apparent excess in the Band 9 flux is only $2 \sigma$, and also note that the case for the NIR nonthermal component is not secure.

Finally, it is worth re-iterating that AT2018cow is a mere $60 \mathrm{Mpc}$ away. The proximity hints at an extensive population of which AT2018cow is the prototype. The key distinction between AT2018cow and other fast transients is the strong millimeter and submillimeter emission. In general, it is apparent from Figure 5 that an energetic shock propagating into a dense medium will exhibit strong millimeter emission during the first weeks. Many other SNe would likely have had bright emission at mm-submm wavelengths, had they been observed early at those wavelengths. Combining velocities measured at very early times at such short wavelengths, with much later observations at low frequencies could reveal the slowing of the shock associated with the transition from free expansion to the Sedov phase, constraining the total energy in relativistic ejecta. Taken together, these two developments, given that we are now squarely in the era of industrial optical time domain astronomy (e.g., PS-1, PS-2, ASAS-SN, ATLAS, ZTF, and soon BlackGEM), argue for a high-frequency facility dedicated to the pursuit of transients.

The code used to produce the results described in this paper was written in Python and is available online in an open-source repository. ${ }^{18}$

The authors are grateful to the staff at the SMA, the CSIRO Astronomy and Space Science (CASS), and ALMA for rapidly scheduling and executing the observations and reducing the data. It is a pleasure to thank John Carpenter for his guidance and his assistance with the ALMA observations. Thank you to Dale Frail, Raffaella Margutti, and Roger Chevalier for providing feedback on the manuscript, and Gregg Hallinan, Dillon Dong, and Jacob Jencson for helpful discussions. Finally, we would like to thank the anonymous referee for thoughtful suggestions that greatly improved the clarity of the paper.

A.Y.Q.H. was supported by a National Science Foundation Graduate Research Fellowship under Grant No. DGE-1144469. This work was supported by the GROWTH project funded by the National Science Foundation under PIRE Grant No. 1545949. This research was funded in part by the Gordon and Betty Moore Foundation through Grant GBMF5076 to E.S.P., and A.Y.Q.H., E.S.P., and S.R.K. benefited from interactions with Dan Kasen, David Khatami, and Eliot Quataert funded by that grant. T.M. acknowledges the support of the Australian Research Council through Grant FT150100099. D.D. is supported by an Australian Government Research Training Program Scholarship.

The SMA is a joint project between the Smithsonian Astrophysical Observatory and the Academia Sinica Institute of Astronomy and Astrophysics and is funded by the Smithsonian Institution and the Academia Sinica. The ATCA is part of the Australia Telescope National Facility, which is funded by the Australian Government for operation as a National Facility managed by CSIRO. This paper makes use of the following ALMA data: ADS/JAO.ALMA\#2017.A.00047. T. ALMA is a partnership of ESO (representing its member states), NSF (USA), and National Institutes of Natural Sciences (NINS) (Japan), together with National Research Council

\footnotetext{
18 https://github.com/annayqho/AT2018cow
} 
(NRC) (Canada), Taiwan Ministry of Science and Technology (MOST), and Academia Sinica Institute of Astronomy and Astrophysics (ASIAA) (Taiwan), and Korea Astronomy and Space Science Institute (KASI) (Republic of Korea), in cooperation with the Republic of Chile. The Joint ALMA Observatory is operated by ESO, Associated Universities, Inc. (AUI)/NRAO and National Astronomical Observatory of Japan (NAOJ). The National Radio Astronomy Observatory is a facility of the National Science Foundation operated under cooperative agreement by Associated Universities, Inc. This work made use of data supplied by the UK Swift Science Data Centre at the University of Leicester.

Software: Astropy (Astropy Collaboration et al. 2013, 2018), IPython (Pérez \& Granger 2007), Matplotlib (Hunter 2007), NumPy (Oliphant 2006), and SciPy (Jones et al. 2001).

\section{Appendix A ALMA Band 9 Calibration}

For ALMA Band 9, due to the relatively low signal-to-noise of the data, all eight spectral windows were combined to derive a combined phase solution, which was then mapped to each individual spectral window. As a result, no in-band analysis of the spectral index was done and a single flux value was derived for the Band 9 imaging. When fitting a Gaussian function to the Band 9 image, the source is represented as a point source, suggesting that the image is of good enough quality to derive a meaningful flux densities. A phase-only self-calibration did not provide good solutions, it decreased the phase coherence, and it resulted in an image that could no longer be fitted with a point source. Therefore, we did not apply a self-calibration to the Band 9 data. To verify that changing weather conditions did not affect the phase coherence in the data, we split the data in three different time bins and imaged each time bin separately. The change in flux density between the different time bins was within 6\%. Similarly, imaging the data from the short, intermediate and long baselines by splitting the data in three bins in the UV range (12-90 $\mathrm{m}, 90-170 \mathrm{~m}$, and $170-312 \mathrm{~m}$ ) showed a difference in flux density $<13 \%$, despite the sparser antenna distribution and poor UV coverage in the long-baseline

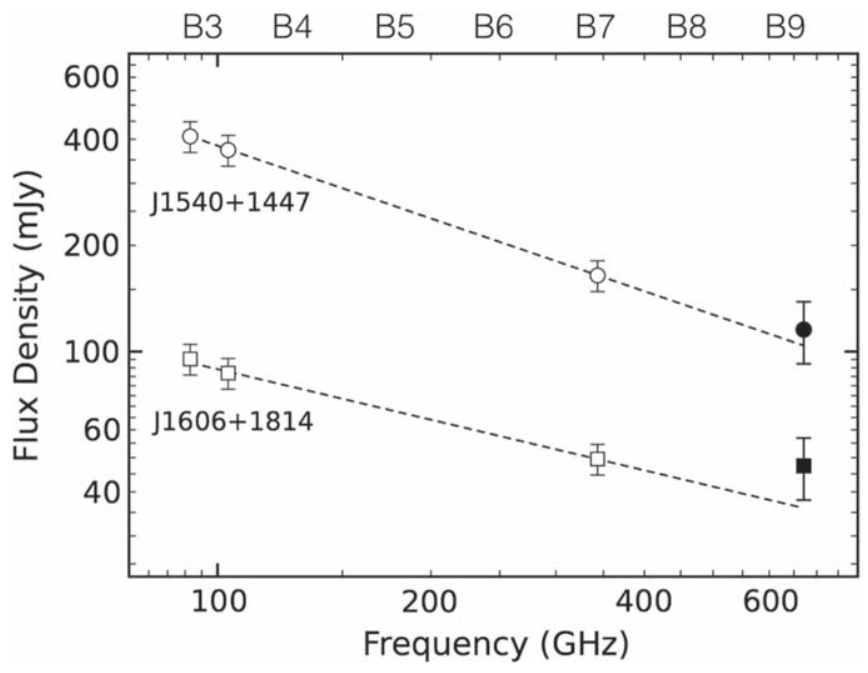

Figure 8. ALMA flux measurements of secondary calibrators J1540+1447 and $\mathrm{J} 1606+1814$, validating the absolute flux calibration of the Band 9 data. The solid symbol shows our ALMA Band 9 measurement on 2018 July 10. The open symbols represent the estimated flux densities in Bands 3 and 7 on July 10 , derived by interpolating between archival values measured by ALMA on 2018 May 18 and August 9. Error bars represent 10\% uncertainties for the archival data and $20 \%$ for our measured Band 9 data. The straight-dashed lines have been added for purpose of visualization and do not represent an actual fit to the data. ALMA bands B3-B9 are shown at the top for reference.

bin. Also, the XX and YY polarization images were similar to within $3 \%$. To examine the reliability of the absolute flux calibration of the Band 9 data, we imaged the two secondary calibrators, the quasars $\mathrm{J} 1540+1447$ and $\mathrm{J} 1606+1814$, using the same flux and bandpass calibrator as for AT2018cow. Figure 8 shows that the Band 9 flux densities of these two secondary calibrators are in reasonable agreement with values from the ALMA calibrator catalog in the lower bands if there is no spectral curvature, although uncertainties in absolute flux calibration may have led us to slightly overpredict our derived Band 9 values. In all, our tests are consistent with the ALMA Band 9 flux density being accurate to within a $20 \%$ uncertainty, which is standard for high-frequency ALMA observations. 

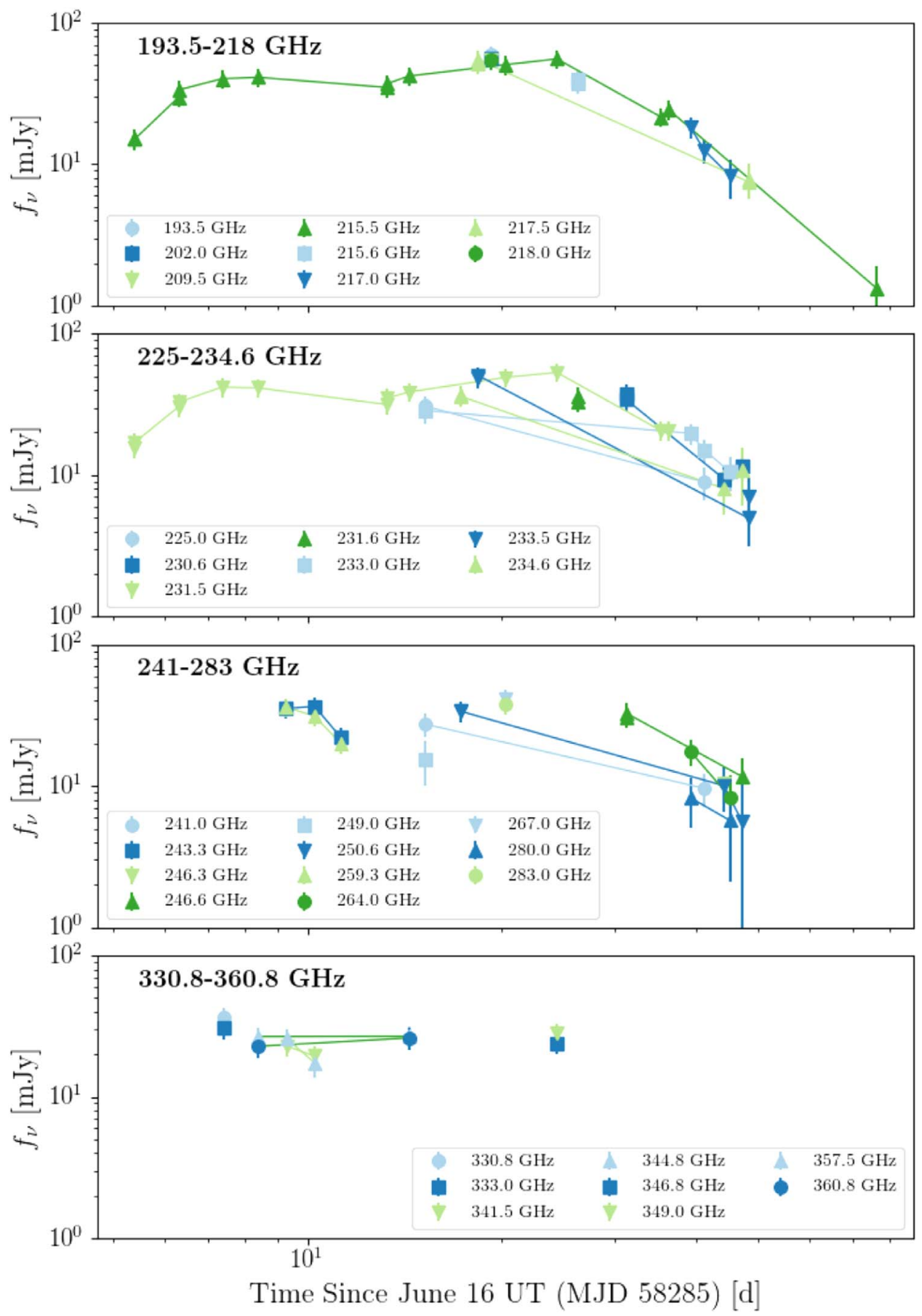

Figure 9. Full SMA light curves of AT2018cow for each individual frequency tuning.

\section{Appendix B \\ Full SMA Light Curves}

Figure 9 shows the full set of SMA light curves, grouped by frequency.

\section{Appendix C}

Selection of Peak Frequency and Peak Luminosity for Other Transients

1. GRB 130427A ( $z=0.340)$ : For Figure 6 , we use the $93 \mathrm{GHz}$ light curve and 5.1 GHz light curve from Perley et al. (2014).
2. GRB $030329(z=0.1686)$ : For Figure 6, we use the $250 \mathrm{GHz}$ light curve at high frequencies (Sheth et al. 2003), and at low frequencies the 8.5 and $2.3 \mathrm{GHz}$ light curves from Berger et al. (2003) and van der Horst et al. (2008) respectively.

3. $S N 2009 b b(d=40 \mathrm{Mpc})$ : For Figures 4 and 5 , we use the report in Soderberg et al. (2010) that from their first spectrum at $\Delta t=20$ days, they infer $\nu_{p}=6 \mathrm{GHz}$ and $L_{p} \approx 3.6 \times 10^{28} \mathrm{erg} \mathrm{s}^{-1} \mathrm{~Hz}^{-1}$. For Figure 6, we use the $8.5 \mathrm{GHz}$ light curve from Soderberg et al. (2010). 
4. $S N 1998 b w(d=38 \mathrm{Mpc})$ : For Figures 4 and 5, we use the report in Kulkarni et al. (1998) that on Day 10 the peak flux is $50 \mathrm{mJy}$ at $10 \mathrm{GHz}$. For Figure 6, we show the single $150 \mathrm{GHz}$ measurement by Submillimetre Common-User Bolometer Array (SCUBA) and the 2.3 GHz light curve from Kulkarni et al. (1998).

5. SN 2006aj $(z=0.03345)$ : For Figures 4 and 5 , we use the report in Soderberg et al. (2006) that at 5 days the radio spectrum peaks near $4 \mathrm{GHz}$. They do not report the peak luminosity, so we use the reported flux of $4.86 \mathrm{GHz}$ at 5 days, which is $328 \mu \mathrm{Jy}$.

6. SN 2010bh $(z=0.0593)$ : For Figures 4 and 5, we use the report in Margutti et al. (2013) that at 30 days, $\nu_{a} \approx 5 \mathrm{GHz}$ and $F_{\nu, a} \approx 130 \mu \mathrm{Jy}$.

7. PTF 11qcj $(z=0.0287)$ : For Figures 4 and 5, we use the report in Corsi et al. (2014) that the peak luminosity at $5 \mathrm{GHz}$ was $7 \times 10^{28} \mathrm{erg} \mathrm{s}^{-1} \mathrm{~Hz}^{-1}$ at 10 days.

8. $S N 2011 d h(d=8.03 \mathrm{Mpc})$ : For Figure 6, we use the 107 and $93 \mathrm{GHz}$ light curves at high frequencies (Horesh et al. 2013) and the 8.5 and $6.7 \mathrm{GHz}$ light curves at low frequencies (Krauss et al. 2012; Horesh et al. 2013).

9. SN 2007bg $(d=152 \mathrm{Mpc})$ : For Figures 4 and 5, we use the report in Salas et al. (2013) that in Phase 1 of the explosion, the peak luminosity was $4.1 \times$ $10^{28} \mathrm{erg} \mathrm{s}^{-1} \mathrm{~Hz}^{-1}$ at $8.46 \mathrm{GHz}$ on Day 55.9. For Figure 6 , we use the $8.5 \mathrm{GHz}$ light curve from Salas et al. (2013).

10. $S N 2003 L(d=92 \mathrm{Mpc})$ : For Figures 4 and 5, we use the report in Soderberg et al. (2005) that at 30 days, the peak flux density was $3.2 \mathrm{mJy}$ at $22.5 \mathrm{GHz}$. For Figure 6, we use the $8.5 \mathrm{GHz}$ light curve because it is the best-sampled over the largest range of time.

11. SN 2003bg $(d=19.6 \mathrm{Mpc})$ : For Figures 4 and 5, we use the report in Soderberg et al. (2006) that the peak flux density is $85 \mathrm{mJy}$ at $22.5 \mathrm{GHz}$ on Day 35. For Figure 6, we use the $8.5 \mathrm{GHz}$ light curve from Soderberg et al. (2006).

12. $S N 1993 J(d=3.63 \mathrm{Mpc})$ : For Figure 6 , we use the $5 \mathrm{GHz}$ light curve at low frequencies and the $99.4 \mathrm{GHz}$ light curve at high frequencies (Weiler et al. 2007).

13. $S N 1988 Z(z=0.022)$ : For Figures 4 and 5 , we use the report in van Dyk et al. (1993) that the $6 \mathrm{~cm}$ maximum flux density was $1.90 \mathrm{mJy}$, at 1253 days after the explosion.

14. SN 1979C (20 Mpc): For Figure 6, we use the $1.4 \mathrm{GHz}$ light curve at low frequencies and the $99.4 \mathrm{GHz}$ light curve at high frequencies (Weiler et al. 1986, 1991). For Figures 4 and 5, we simply use the peak of the $1.4 \mathrm{GHz}$ light curve, which is roughly $12 \mathrm{mJy}$ at 1400 days.

15. Swift J1644+57 $(z=0.354)$ : For Figures 4 and 5, we use the reported $\nu_{p}, F_{p}$ on Day 15 (corrected to Day 18 in Eftekhari et al. 2018). For Figure 6, we use the 225 and $230 \mathrm{GHz}$ light curves from the SMA (Zauderer et al. 2011; Berger et al. 2012), adding 3.04 days to the Zauderer et al. (2011) points because (as described in Eftekhari et al. 2018) subsequent analysis of the BAT data revealed emission earlier than had been previously noticed. We use 4.9 GHz data from Berger et al. (2012), Zauderer et al. (2013), and Eftekhari et al. (2018).

\section{ORCID iDs}

Anna Y. Q. Ho (iD https://orcid.org/0000-0002-9017-3567 Vikram Ravi (i) https://orcid.org/0000-0002-7252-5485 S. R. Kulkarni iD https://orcid.org/0000-0001-5390-8563 V. Bhalerao (ii) https://orcid.org/0000-0002-6112-7609

S. Bradley Cenko (1) https://orcid.org/0000-0003-1673-970X

Dougal Dobie (iD https://orcid.org/0000-0003-0699-7019

Nikita Kamraj (iD https://orcid.org/0000-0002-3233-2451

Mansi M. Kasliwal (i) https://orcid.org/0000-0002-5619-4938

Tara Murphy (iD https://orcid.org/0000-0002-2686-438X

Daniel A. Perley (i) https://orcid.org/0000-0001-8472-1996

Ilsang Yoon (D) https://orcid.org/0000-0001-9163-0064

\section{References}

Alexander, K. D., Berger, E., Guillochon, J., Zauderer, B. A., \& Williams, P. K. G. 2016, ApJL, 819, L25

Astropy Collaboration, Price-Whelan, A. M., Sipőcz, B. M., et al. 2018, AJ, 156,123

Astropy Collaboration, Robitaille, T. P., Tollerud, E. J., et al. 2013, A\&A, 558, A33

Berger, E., Kulkarni, S. R., Pooley, G., et al. 2003, Natur, 426, 154

Berger, E., Zauderer, A., Pooley, G. G., et al. 2012, ApJ, 748, 36

Blandford, R., \& Eichler, D. 1987, PhR, 154, 1

Bower, G. C., Plambeck, R. L., Bolatto, A., et al. 2003, ApJ, 598, 1140

Bright, J., Horesh, A., Fender, R., et al. 2018, ATel, 11774

Bucciantini, N., Quataert, E., Arons, J., Metzger, B. D., \& Thompson, T. A. 2007, MNRAS, 380, 1541

Burrows, D. N., Hill, J. E., Nousek, J. A., et al. 2005, SSRv, 120, 165

Chevalier, R. A. 1982, ApJ, 259, 302

Chevalier, R. A. 1983, ApJ, 272, 765

Chevalier, R. A. 1984, NYASA, 422, 215

Chevalier, R. A. 1998, ApJ, 499, 810

Chevalier, R. A., \& Fransson, C. 2006, ApJ, 651, 381

Corsi, A., Ofek, E. O., Gal-Yam, A., et al. 2014, ApJ, 782, 42

Dal Canton, T., Briggs, M. S., Burns, E., et al. 2018, ATel, 11793

de Kool, M., Begelman, M. C., \& Sikora, M. 1989, ApJ, 337, 66

Dent, W. A., O’Dea, C. P., Balonek, T. J., Hobbs, R. W., \& Howard, R. J. 1983, Natur, 306, 41

de Ugarte Postigo, A., Bremer, M., Kann, D. A., et al. 2018, ATel, 11749 de Ugarte Postigo, A., Lundgren, A., Martín, S., et al. 2012, A\&A, 538, A44 Dobie, D., Ravi, V., Ho, A., Kasliwal, M., \& Murphy, T. 2018a, ATel, 11795 Dobie, D., Ravi, V., Ho, A., Kasliwal, M., \& Murphy, T. 2018b, ATel, 11818 Draine, B. T. 2011, Physics of the Interstellar and Intergalactic Medium (Princeton, NJ: Princeton Univ. Press)

Drout, M. R., Chornock, R., Soderberg, A. M., et al. 2014, ApJ, 794, 23

Eftekhari, T., Berger, E., Zauderer, B. A., Margutti, R., \& Alexander, K. D. 2018, ApJ, 854, 86

Evans, P. A., Beardmore, A. P., Page, K. L., et al. 2009, MNRAS, 397, 1177 Fender, R. P., Anderson, G. E., Osten, R., et al. 2015, MNRAS, 446, L66

Ferrigno, C., Kuulkers, E., Goetz, D., et al. 2018, ATel, 11788

Frail, D. A., Waxman, E., \& Kulkarni, S. R. 2000, ApJ, 537, 191

Frater, R. H., Brooks, J. W., \& Whiteoak, J. B. 1992, JEEEA, 12, 103 Fremling, C. 2018, ATel, 11738

Gehrels, N., Chincarini, G., Giommi, P., et al. 2004, ApJ, 611, 1005

Granot, J., \& Sari, R. 2002, ApJ, 568, 820

Grefenstette, B., Margutti, R., Chornock, R., Metzger, B., \& Migliori, G. 2018, ATel, 11813

Guilbert, P. W. 1986, MNRAS, 218, 171

Harrison, F. A., Craig, W. W., Christensen, F. E., et al. 2013, ApJ, 770, 103

Herczeg, G. J., Johnstone, D., Mairs, S., et al. 2017, ApJ, 849, 43

Ho, P. T. P., Moran, J. M., \& Lo, K. Y. 2004, ApJL, 616, L1

Horesh, A., Stockdale, C., Fox, D. B., et al. 2013, MNRAS, 436, 1258

Hunter, J. D. 2007, CISE, 9, 90

Jencson, J. E., Kasliwal, M. M., Adams, S. M., et al. 2018, ApJ, 863, 20

Jones, E., Oliphant, E., \& Peterson, P. 2001, SciPy: Open Source Scientific Tools for Python, http://www.scipy.org/

Jones, F. C., \& Ellison, D. C. 1991, SSRv, 58, 259

Kasen, D., \& Bildsten, L. 2010, ApJ, 717, 245

Kasen, D., Metzger, B. D., \& Bildsten, L. 2016, ApJ, 821, 36

Kaspi, V. M., Archibald, R. F., Bhalerao, V., et al. 2014, ApJ, 786, 84 
Kocevski, D., \& Cheung, C. C. 2018, ATel, 11808

Krauss, M. I., Soderberg, A. M., Chomiuk, L., et al. 2012, ApJL, 750, L40

Kuin, N. P. M., Wu, K., Oates, S., et al. 2018, arXiv:1808.08492

Kulkarni, S. R., Frail, D. A., Wieringa, M. H., et al. 1998, Natur, 395, 663

Kumar, P., \& Barniol Duran, R. 2010, MNRAS, 409, 226

Kumar, P., \& Zhang, B. 2015, PhR, 561, 1

Lamers, H. J. G. L. M., \& Leitherer, C. 1993, ApJ, 412, 771

Lang, K. R. 1999, Astrophysical Formulae (New York: Springer)

Laskar, T., Berger, E., Zauderer, B. A., et al. 2013, ApJ, 776, 119

Lien, A. Y., Palmer, D. M., Tohuvavohu, A., et al. 2018, ATel, 11782

Lundqvist, P., \& Fransson, C. 1988, A\&A, 192, 221

Madau, P., \& Efstathiou, G. 1999, ApJL, 517, L9

Margutti, R., Chornock, R., Laskar, T., et al. 2018a, ATel, 11775

Margutti, R., Metzger, B. D., Chornock, R., et al. 2018b, arXiv:1810.10720

Margutti, R., Soderberg, A. M., Wieringa, M. H., et al. 2013, ApJ, 778, 18

McMullin, J. P., Waters, B., Schiebel, D., Young, W., \& Golap, K. 2007, adass XVI, 376, 127

Michel, F. C. 1988, Natur, 333, 644

Middelberg, E., Sault, R. J., \& Kesteven, M. J. 2006, PASA, 23, 147

Nakar, E. 2015, ApJ, 807, 172

Nakar, E., \& Piran, T. 2011, Natur, 478, 82

Ofek, E. O., Rabinak, I., Neill, J. D., et al. 2010, ApJ, 724, 1396

Oliphant, T. E. 2006, Guide to NumPy (Scotts Valley, CA: CreateSpace Independent Publishing Platform)

Pacholczyk, A. G. 1970, Series of Books in Astronomy and Astrophysics (San Francisco: Freeman)

Pelletier, G., Bykov, A., Ellison, D., \& Lemoine, M. 2017, SSRv, 207, 319

Pérez, F., \& Granger, B. E. 2007, CISE, 9, 29

Perley, D. A., Cenko, S. B., Corsi, A., et al. 2014, ApJ, 781, 37

Perley, D. A., Mazzali, P. A., Yan, L., et al. 2018, arXiv:1808.00969

Phinney, E. S. 1989, in IAU Symp. 136, The Center of the Galaxy 136, ed.

M. Morris (Dordrecht: Kluwer Academic Publishers), 543

Prentice, S. J., Maguire, K., Smartt, S. J., et al. 2018, ApJL, 865, L3

Pursiainen, M., Childress, M., Smith, M., et al. 2018, MNRAS, 481, 894

Readhead, A. C. S. 1994, ApJ, 426, 51

Rest, A., Garnavich, P. M., Khatami, D., et al. 2018, NatAs, 2, 307

Rivera Sandoval, L. E., \& Maccarone, T. 2018, ATel, 11737

Rivera Sandoval, L. E., Maccarone, T. J., Corsi, A., et al. 2018, MNRAS, 480, L146

Roming, P. W. A., Kennedy, T. E., Mason, K. O., et al. 2005, SSRv, 120, 95

Rybicki, G. B., \& Lightman, A. P. 1986, Radiative Processes in Astrophysics (New York: Wiley-VCH), 400
Salas, P., Bauer, F. E., Stockdale, C., \& Prieto, J. L. 2013, MNRAS, 428, 1207

Sánchez-Ramírez, R., Hancock, P. J., Jóhannesson, G., et al. 2017, MNRAS, 464, 4624

Sari, R., Piran, T., \& Narayan, R. 1998, ApJL, 497, L17

Sault, R. J., Teuben, P. J., \& Wright, M. C. H. 1995, adass IV, 77, 433

Savchenko, V., Ferrigno, C., Kuulkers, E., et al. 2018, ATel, 11843

Sazonov, S. Y., Ostriker, J. P., \& Sunyaev, R. A. 2004, MNRAS, 347, 144

Sharma, Y., Bhalerao, V., Bhattacharya, D., Rao, A. R., \& Vadawale, S. 2018, ATel, 11809

Sheth, K., Frail, D. A., White, S., et al. 2003, ApJL, 595, L33

Smartt, S. J., Clark, P., Smith, K. W., et al. 2018, ATel, 11727

Smith, I. A., Tanvir, N. R., \& Perley, D. A. 2018, ATel, 11781

Smith, N., Cenko, S. B., Butler, N., et al. 2012, MNRAS, 420, 1135

Smith, N., Kilpatrick, C. D., Mauerhan, J. C., et al. 2017, MNRAS, 466, 3021

Soderberg, A. M., Chakraborti, S., Pignata, G., et al. 2010, Natur, 463, 513

Soderberg, A. M., Chevalier, R. A., Kulkarni, S. R., \& Frail, D. A. 2006, ApJ, 651,1005

Soderberg, A. M., Kulkarni, S. R., Berger, E., et al. 2004, Natur, 430, 648

Soderberg, A. M., Kulkarni, S. R., Berger, E., et al. 2005, ApJ, 621, 908

Thompson, T. A., Chang, P., \& Quataert, E. 2004, ApJ, 611, 380

Tonry, J. L., Denneau, L., Heinze, A. N., et al. 2018, PASP, 130, 064505

Urata, Y., Huang, K., Takahashi, S., et al. 2014, ApJ, 789, 146

Urošević, D. 2014, Ap\&SS, 354, 541

van der Horst, A. J., Kamble, A., Resmi, L., et al. 2008, A\&A, 480, 35

van Dyk, S. D., Weiler, K. W., Sramek, R. A., \& Panagia, N. 1993, ApJL, 419, L69

van Loon, J. T. 2010, in ASP Conf. Ser. 425, Hot and Cool:Bridging Gaps in Massive Star Evolution 425, ed. C. Lietherer et al. (San Francisco, CA: ASP), 279

Weiler, K. W., Sramek, R. A., Panagia, N., van der Hulst, J. M., \& Salvati, M. 1986, ApJ, 301, 790

Weiler, K. W., van Dyk, S. D., Panagia, N., Sramek, R. A., \& Discenna, J. L. 1991, ApJ, 380, 161

Weiler, K. W., Williams, C. L., Panagia, N., et al. 2007, ApJ, 671, 1959

Whitehorn, N., Natoli, T., Ade, P. A. R., et al. 2016, ApJ, 830, 143

Wilson, W. E., Ferris, R. H., Axtens, P., et al. 2011, MNRAS, 416, 832

Yuan, Q., Wang, Q. D., Lei, W.-H., Gao, H., \& Zhang, B. 2016, MNRAS, 461, 3375

Zauderer, B. A., Berger, E., Margutti, R., et al. 2013, ApJ, 767, 152

Zauderer, B. A., Berger, E., Soderberg, A. M., et al. 2011, Natur, 476, 425 